\title{
Les températures extrêmes de l'année 2003 dans le Nord-Est français et ses bordures luxembourgeoise et allemande
}

Extreme air temperatures of 2003 in North-Eastern France and bordering regions Extreme Tageslufttemperaturen des Jahres 2003 im Nordosten Frankreichs und in den Grenzgebieten

Gilles Drogue, Lucien Hoffmann, Laurent Pfister et Patrice Paul

\section{(2) OpenEdition \\ Journals}

Édition électronique

URL : http://journals.openedition.org/rge/451

ISSN : 2108-6478

\section{Éditeur}

Association des géographes de l'Est

\section{Édition imprimée}

Date de publication : 1 avril 2005

Pagination : 79-98

ISSN : 0035-3213

\section{Référence électronique}

Gilles Drogue, Lucien Hoffmann, Laurent Pfister et Patrice Paul, « Les températures extrêmes de l'année 2003 dans le Nord-Est français et ses bordures luxembourgeoise et allemande », Revue Géographique de l'Est [En ligne], vol. 45 / 2 | 2005, mis en ligne le 10 juin 2009, consulté le 07 septembre 2020. URL : http://journals.openedition.org/rge/451

Ce document a été généré automatiquement le 7 septembre 2020

Tous droits réservés 


\title{
Les températures extrêmes de l'année 2003 dans le Nord-Est français et ses bordures luxembourgeoise et allemande
}

\author{
Extreme air temperatures of 2003 in North-Eastern France and bordering regions \\ Extreme Tagesluftemperaturen des Jahres 2003 im Nordosten Frankreichs und \\ in den Grenzgebieten
}

Gilles Drogue, Lucien Hoffmann, Laurent Pfister et Patrice Paul

\section{NOTE DE L'ÉDITEUR}

Article reçu le 15 janvier 2005, accepté le 28 février 2005

La réalisation de cette étude a bénéficié de la fourniture de données françaises via la Climathèque de Météo-France, de données luxembourgeoises via le service météorologique de l'aéroport de Luxembourg (MM. Kirpach et Alesch) et de données allemandes via le Deutscher Wetterdienst. Le financement a été assuré par le projet CLIMAT du programme VIVRE du Fonds National de la Recherche du Grand-Duché de Luxembourg.

1 L'année 2003 restera marquée dans les annales climatologiques par une anomalie thermique positive de grande amplitude affectant toute l'Europe occidentale et occasionnant un stress écologique et sociétal aigu. Son caractère hors-norme offre un double intérêt climatologique :

- D'une part la douceur de l'année 2003 en Europe occidentale, essentiellement imputable à la saison estivale (juin-juillet-août), est sans précédent depuis que des observations instrumentales systématiques et normalisées ont été entreprises par les services météorologiques européens, à partir de la deuxième moitié du XIX siècle. À titre d'exemple, l'analyse de la température moyenne estivale mesurée à Bâle de 1864 à 2003, amène Schär et 
al. (2004) à conclure que l'été 2003 se situe à $5 \sigma$ de la température moyenne estivale de la période 1864-2000, et sort donc largement de la variabilité climatique à laquelle est soumise l'Europe occidentale depuis 150 ans. Le premier objectif de cet article consiste donc à positionner l'année 2003 dans la séquence des années chaudes de la décennie 1990 et par rapport à la dernière normale climatique, pour caractériser l'ampleur de l'élévation des températures à l'échelle de la région Nord-Est et de ses bordures luxembourgeoise et allemande, qui semble s'accélérer au cours de cette période.

- D'autre part, la convergence entre la fonction de densité de probabilité des températures maximales observées en Suisse au cours de la saison estivale de l'année 2003 et les projections climatiques à l'horizon 2070-2099 de certains modèles climatiques de mésoéchelle pour cette même saison, incitent à penser que la signature thermique de l'été 2003 serait une préfiguration des étés qu'expérimentera fréquemment l'Europe occidentale à la fin du siècle sous l'action d'un effet de serre additionnel anthropogénique (Beniston, 2004). Le second objectif de cet article est donc de documenter la distribution spatio-temporelle locale et régionale des températures extrêmes au cours de l'année 2003 et en particulier de la saison estivale dans le Nord-Est français et ses bordures luxembourgeoise et allemande, en prenant en compte l'influence des déterminants géographiques (occupation des sols, topographie) ; enfin il s'agit de proposer une image prospective des étés que connaîtront les générations futures si les scénarios climatiques européens se vérifient.

\section{La zone d'étude}

Bordé à l'ouest par la Meuse et à l'est par le Rhin, le territoire considéré couvre approximativement $50000 \mathrm{~km}^{2}$ et comprend des unités de relief variées comme les reliefs de côte de Lorraine, les massifs vosgien et ardennais ou encore la plaine d'Alsace (figure 1). La disposition et les caractéristiques des massifs montagneux, au premier rang desquels figure le massif vosgien, induisent une dégradation brutale du climat semi-océanique du plateau lorrain relayé vers l'est par un climat tempéré encore semiocéanique, mais où la continentalité s'exprime avec plus de vigueur. Les principales lignes de relief favorisent également l'émergence d'une marqueterie de climats locaux par le jeu des nuances topoclimatiques (Paul, 1997). 
Figure 1 : Orographie de la zone d'étude et réseau de mesure thermométrique utilisé.

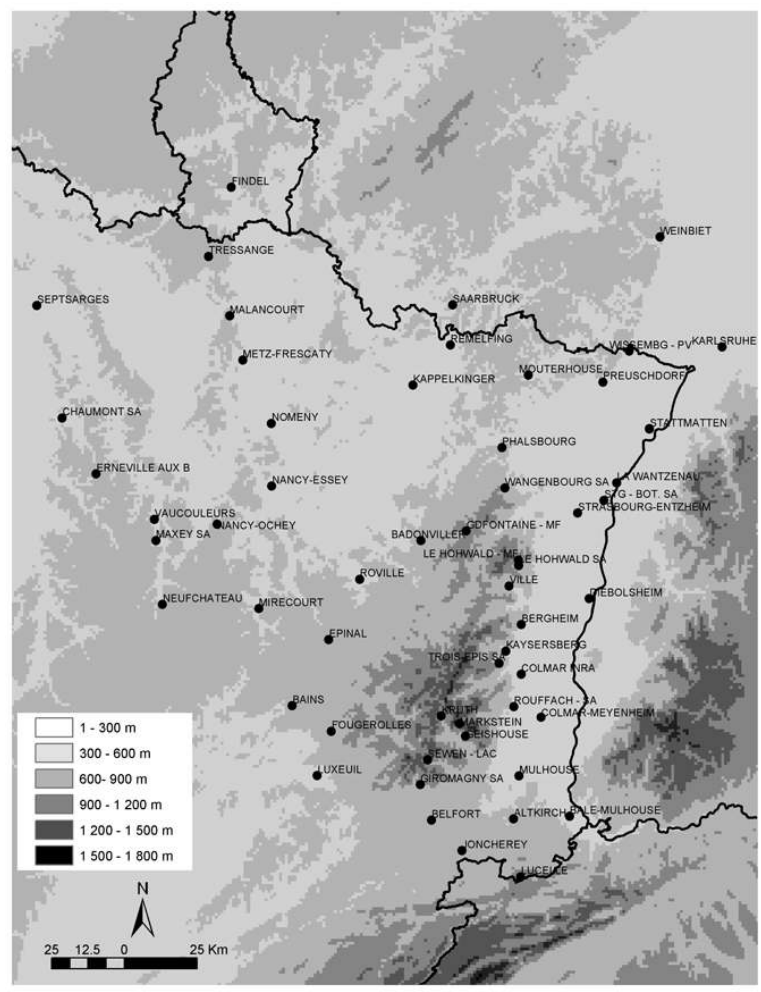

\section{Les grands traits climatiques de l'année 2003}

Selon le bilan climatique publié par Météo-France pour la France (http:// www.meteofrance.com/FR/climat/bilan2003.jsp; Bessemoulin et al., 2004), l'année 2003, exceptionnellement chaude, égale le record précédemment établi en 1994 avec une température moyenne annuelle de $13^{\circ} \mathrm{C}$. De plus, avec $17,9^{\circ} \mathrm{C}$, la moyenne des températures maximales dépasse très largement le précédent record de $17,4^{\circ} \mathrm{C}$ datant de 1989. Toujours selon Météo-France, seuls les mois de janvier, février et octobre sont plus froids que la normale. L'été 2003 est exceptionnellement chaud et marqué par la canicule qui a sévi durant la première quinzaine d'août. Cependant, le mois de juin, avec un écart de $+4,7^{\circ} \mathrm{C}$ par rapport à la normale 1971-2000, enregistre l'excédent le plus important devant août $\left(+4,4^{\circ} \mathrm{C}\right)$ et juillet $\left(+1,8^{\circ} \mathrm{C}\right)$. Corollaire de ces températures exceptionnellement élevées et d'un ensoleillement excédentaire par rapport à la moyenne 1991-2000 et tout particulièrement dans le quart Nord-Est (20 à $30 \%$ audessus de la normale), le nombre annuel de jours pluvieux accuse un déficit de 40 à 50 jours, tandis que le cumul annuel est situé entre 50 et $75 \%$ des valeurs de la normale 1971-2000 dans le Nord-Est français. 


\section{Données et méthodes utilisées}

\section{A. La base de données de température}

4 Les données quotidiennes de températures minimales ( $\mathrm{Tn}$ ) et maximales (Tx) mesurées sous abri ont été collectées à l'échelle du Nord-Est français, principalement à la faveur du service internet d'accès aux données et produits climatologiques de Météo-France (Climathèque), qui donne la possibilité de rapatrier en ligne un grand nombre de paramètres météorologiques à différents pas de temps. Outre la fourniture de séries de données brutes, l'application Climathèque renseigne également l'utilisateur sur le type de station de mesure, sa localisation et son historique. L'ensemble de ces métadonnées facilite considérablement l'interprétation des conclusions analytiques, comme nous le verrons plus loin.

5 Des stations climatologiques périphériques ont été ajoutées au réseau de mesure strictement français pour éviter les effets de bord frontaliers lors de la génération des cartes de températures annuelles. Ainsi, des stations allemandes et luxembourgeoises ont été prises en compte, l'ensemble du réseau de mesure mobilisé in fine comportant 56 stations de mesure dont 53 postes français (figure 1 et annexe A), répartis en trois groupes de 25 stations sur le plateau lorrain, 8 stations sur le massif vosgien stricto sensu et 20 stations situées en plaine d'Alsace ou en position de piémont vosgien ou jurassien.

6 Précisons que parmi l'ensemble des stations de mesure ayant fonctionné en 2003 et disponibles via Climathèque, seules celles dotées des séries quotidiennes de températures extrêmes continues au cours de l'année 2003 ont été retenues, qualité également valable pour les trois séries de Tn et Tx limitrophes. Il convient de préciser par ailleurs que le type de station tel qu'il figure dans le tableau de l'annexe A correspond au type le plus récent et ne rend pas compte de l'historique complet des paramètres mesurés à la station de mesure. C'est pourquoi, par exemple, la température de l'air n'a été prise en compte qu'à partir de 1994 au Markstein (année de la mise en place de la station automatique), alors que l'ouverture de la station date de 1989 (poste climatologique).

7 Les caractéristiques de site de chacune des stations de mesure ont été consignées systématiquement dans le but de mieux comprendre les effets locaux sous-jacents à la distribution spatiale de températures extrêmes au cours de l'été 2003 (annexe B). La situation topographique et l'environnement des stations de mesure françaises ont été définis grâce aux extraits de fond de plan topographique IGN sur lesquels le service Climathèque de Météo-France reporte systématiquement la position des sites de mesure. Trois exemples caractéristiques sont donnés à titre illustratif (figures $2 a, 2 b$ et 2c). 
Figure 2a :Site de pied de versant en fond de vallon et forestier (Badonviller).

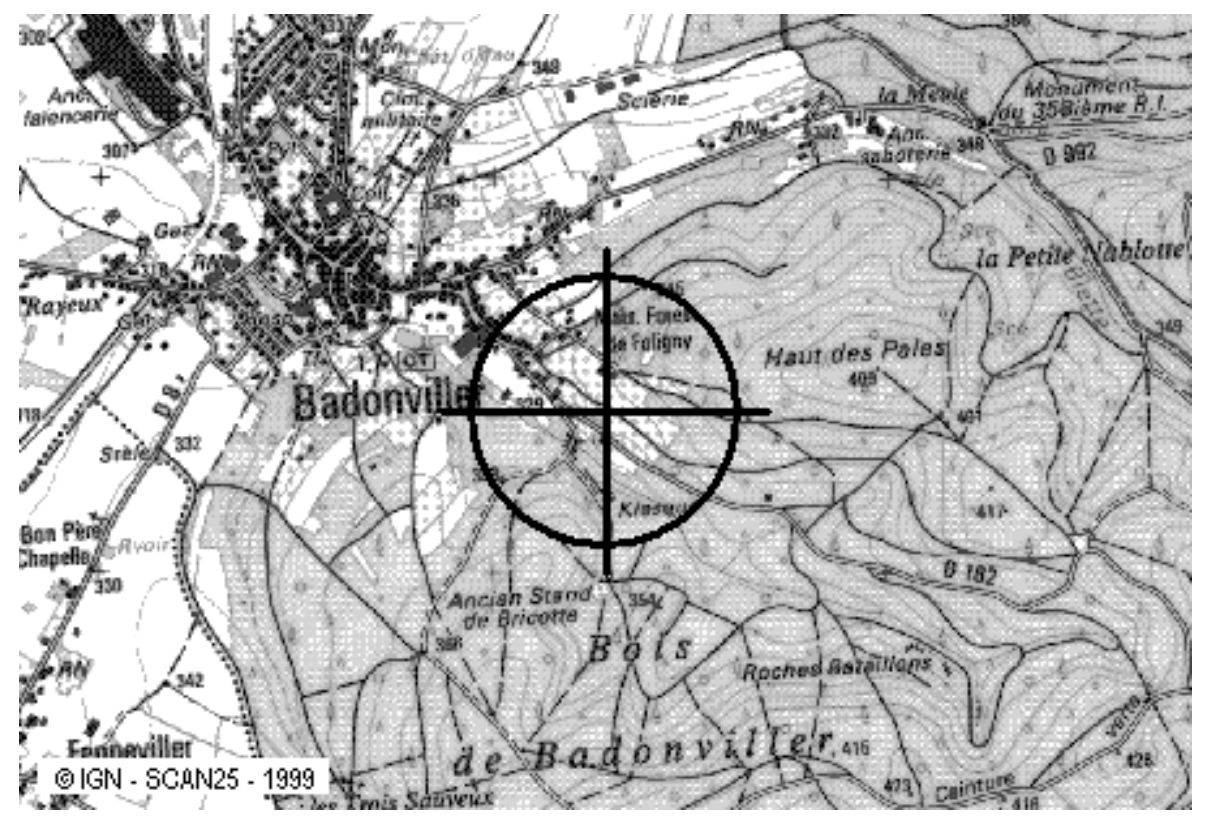

D’après les renseignements fournis par le service Climathèque de Météo-France.

Figure $2 b$ : Site de pied de versant en fond de vallée, suburbain avec présence d'eau (Remelfing).

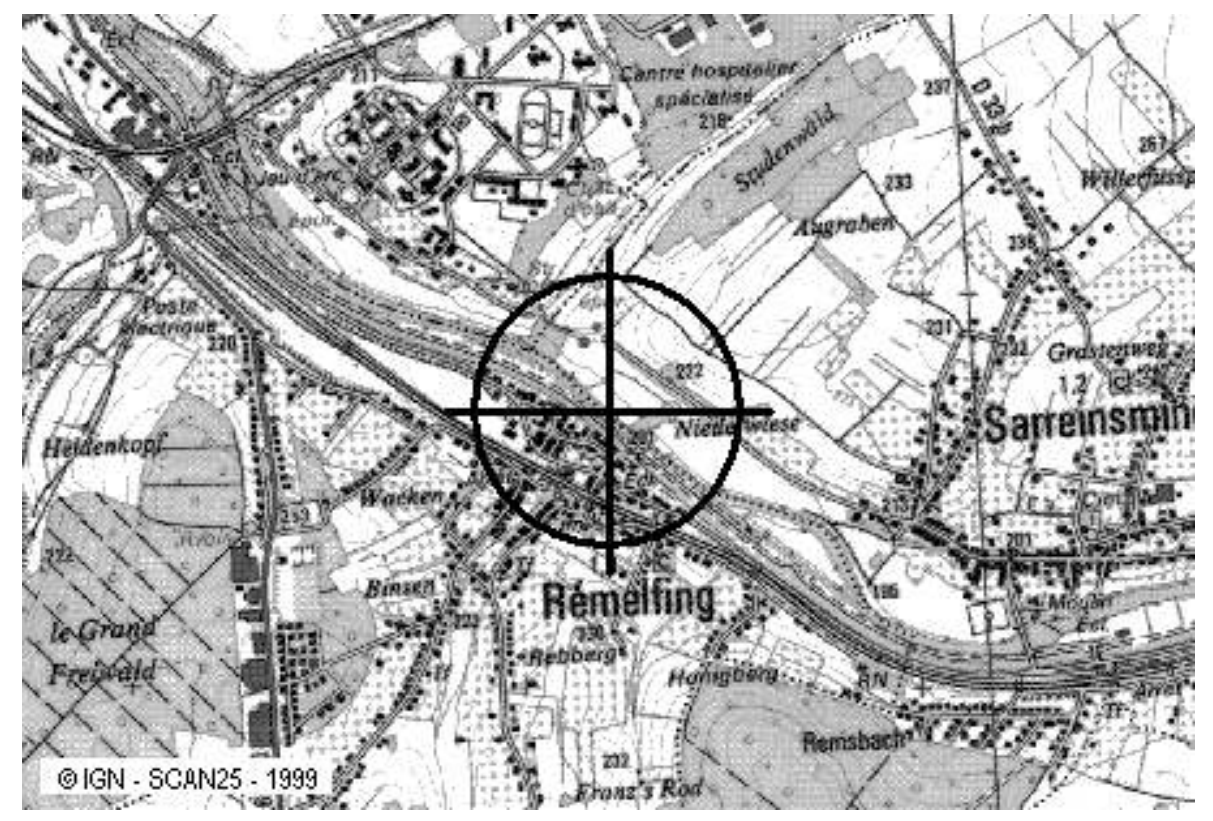

D’après les renseignements fournis par le service Climathèque de Météo-France. 
Figure 2c : Site de fond de vallée ample dans un tissu urbain lâche (Belfort).

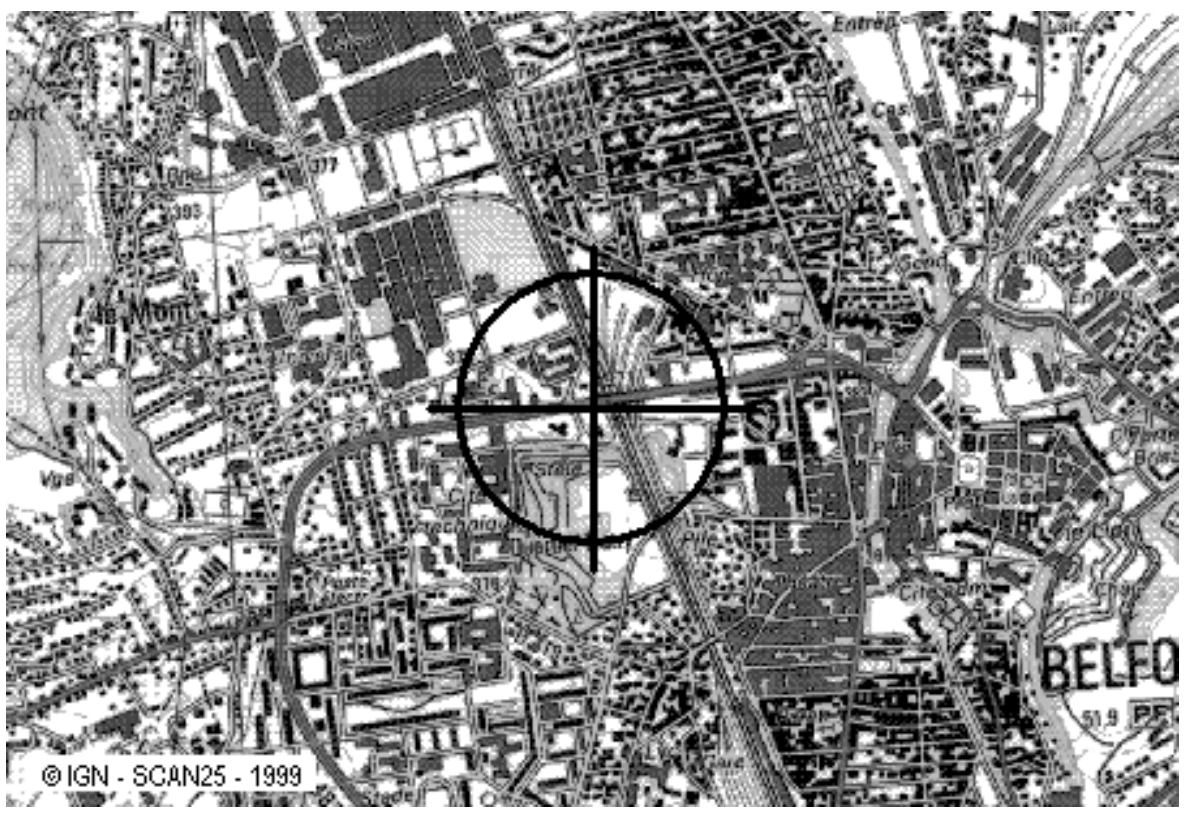

D'après les renseignements fournis par le service Climathèque de Météo-France.

8 Notons que l'attribution d'un type d'environnement à une station de mesure donnée est assez subjective, en particulier lorsqu'il s'agit d'évaluer l'impact d'une agglomération sur le climat local ; par exemple, l'effet d'ilôt de chaleur urbain varie en fonction de la taille et de la densité d'une agglomération (Oke, 1987). Certains climatologues comme Hansen et al. (2001) exploitant les résultats de Imhoff et al. (1997), s'appuient sur la brillance des images satellitales et l'éclairage nocturne émis par les agglomérations pour qualifier l'environnement de leurs stations de mesure. En l'absence d'une telle information sur notre région d'étude, la typologie à 5 classes des environnements des stations (aérodrome/aéroport, site forestier, site sub-urbain, site urbain, site rural) s'est fondée sur un critère de proximité et de "dominance » d'un thème d'occupation des sols. Le distinguo urbain/sub-urbain par exemple, repose sur la localisation centrale ou périphérique de la station de mesure et de l'importance des surfaces minérales environnantes susceptibles de modifier de manière importante le bilan d'énergie local (figure $2 \mathrm{~b}$ versus $2 \mathrm{c}$ ).

9 Il ressort de la figure 3 qu'une majorité de stations de mesure sont localisées dans un environnement rural. L'exposition de chacune des stations de mesure (annexe B) a été déterminée à l'aide du MNT de résolution $1 \mathrm{~km} \times 1 \mathrm{~km}$ de la Figure 1 et validée par référence aux extraits de fond de plan topographique IGN précités. L'examen de l'étagement de stations de mesure par classe d'altitude (figure 4) permet de se rendre compte que la majorité d'entre elles est située en dessous de $500 \mathrm{~m}$, avec une altitude modale entre 200 et $300 \mathrm{~m}$, et qu'un hiatus important apparait entre 800 et $1100 \mathrm{~m}$ d'altitude, la station du Markstein à $1184 \mathrm{~m}$ étant le seul point de mesure en position de crête sommitale dans le massif vosgien. La ventilation des stations de mesure par classe d'exposition révèle une prédominance des sites d'adret, avec deux classes majeures d'orientation sud-ouest et sud-est, toute la gamme d'expositions étant néanmoins représentée (figure 5), ce qui a son importance d'un point de vue topoclimatique. Enfin, les stations de mesure sont préférentiellement implantées en pied de versant ou en zone de plaine (annexe A). 
Figure 3 : Typologie des stations de mesure thermométrique en fonction de leur environnement.

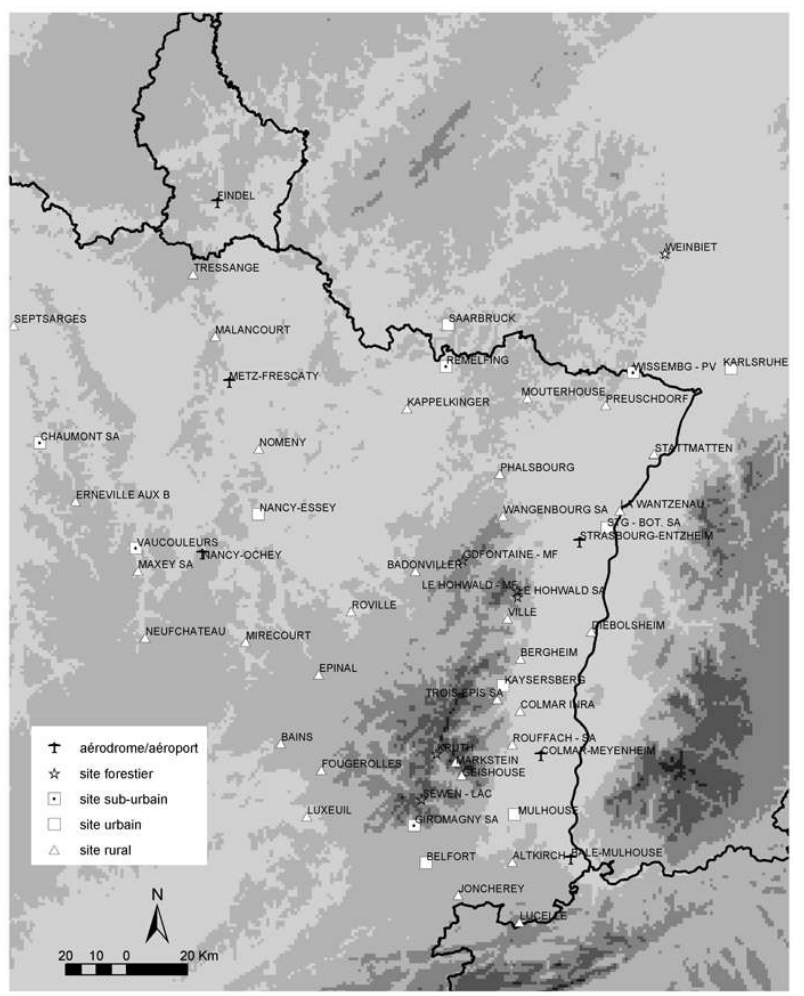

Figure 4 :Répartition des stations de mesure par tranche d'altitude.

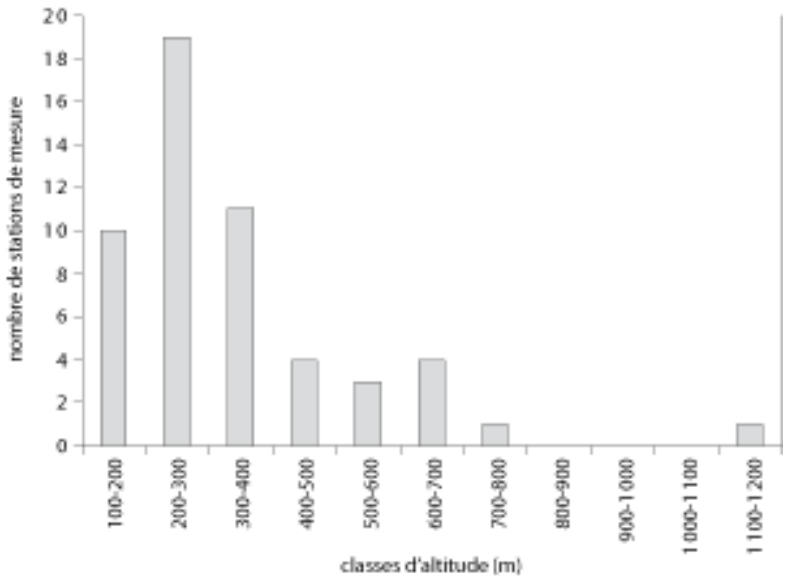


Figure 5 : Répartition en pourcentage des stations de mesure par orientation cardinale.

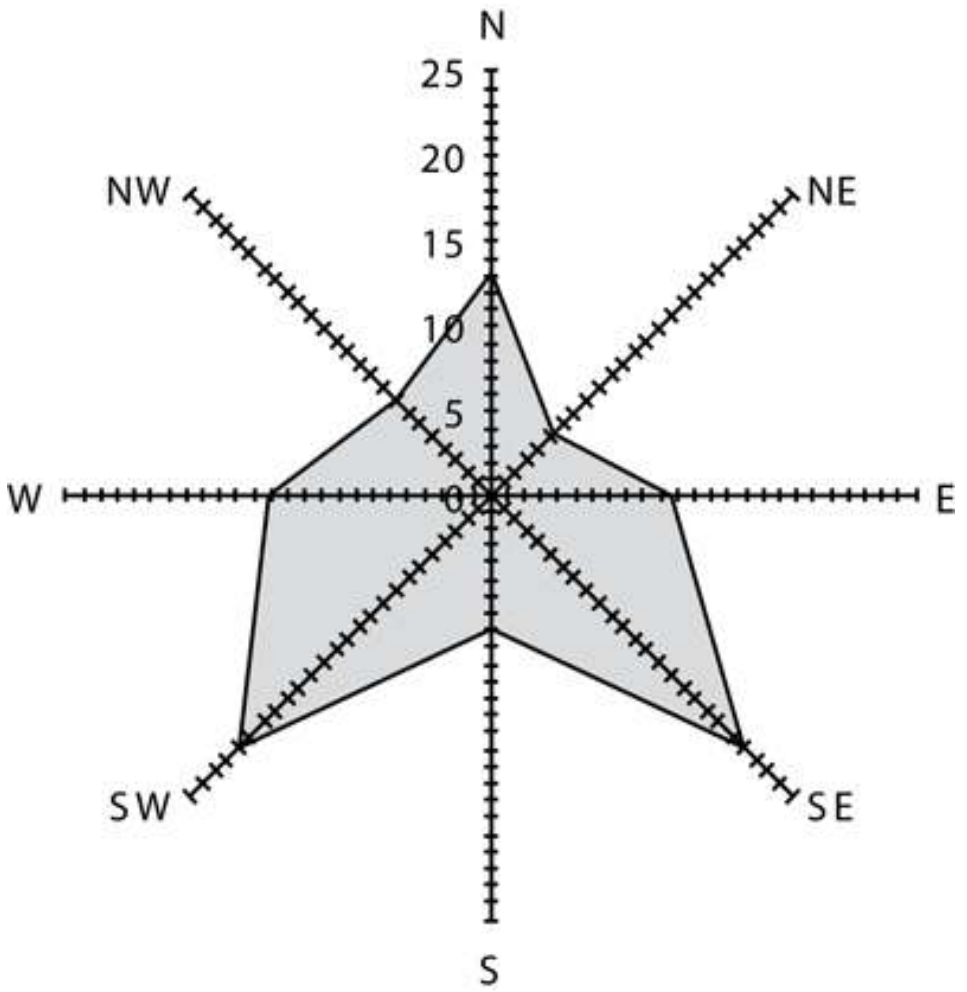

\section{B. Critique des données de température}

Les séries quotidiennes de températures extrêmes afférentes aux 56 stations de mesure ont fait l'objet, dans un premier temps, d'une visualisation graphique systématique par lot de stations voisines pour vérifier la bonne co-fluctuation de leurs courbes de température. Celles-ci ont été soumises ensuite à deux tests logiques destinés à détecter d'éventuelles valeurs aberrantes ou douteuses: d'une part nous avons vérifié que l'ensemble des valeurs quotidiennes de Tn $(18 \mathrm{~h}$ TU jour $\mathrm{J}$ à $18 \mathrm{~h}$ TU jour $\mathrm{J}+1)$ étaient supérieures à $-50^{\circ} \mathrm{C}$ et que celles de Tx $(6 \mathrm{~h}$ TU jour $\mathrm{J}$ à $6 \mathrm{~h} \mathrm{TU}$ jour $\mathrm{J}+1)$ étaient inférieures à $+50^{\circ} \mathrm{C}$; d'autre part, nous avons vérifié l'absence de valeurs de température rémanentes $(T(j)=T(j+1)=T(j+2))$ physiquement peu probables et liées par exemple à un problème de transcription au sein des séries de données.

Sur l'ensemble du jeu de données brutes collectées initialement pour les 56 stations de mesure, deux séries quotidiennes 2003 lacunaires afférentes à Badonviller (1 valeur de Tx manquante) et Le Hohwald SA (14 valeurs de Tn et Tx manquantes) ont été identifiées. Les valeurs manquantes n'ont pas été reconstituées pour deux raisons : i) le nombre de valeurs manquantes n'est pas suffisant pour modifier les moyennes annuelles; ii) les dates d'occurrence des valeurs manquantes se situent en dehors des périodes de calcul des indices de température (cf. infra). Aucune autre valeur de température n'a finalement été rejetée à l'issue de la critique des données.

L'objectif de notre étude étant de positionner l'année 2003 par rapport à des moyennes caractéristiques, décennale et trentenaire, un jeu de séries de températures extrêmes mensuelles et annuelles sans lacunes et intangibles ont été extraites de la base de 
données Climathèque pour la décennie 1991-2000 (46 stations françaises) et la dernière normale climatique standard 1971-2000 (12 stations françaises) (annexe A). L'intangibilité de ces stations, qui n'ont donc pas subi de changement de site au cours de la période étudiée, garantit en première instance l'homogénéité des séries de mesure.

\section{Définition et cartographie des indices de température} 2003 et plus spécifiquement de l'été 2003, différents indices de température ont été quantifiés à partir des valeurs quotidiennes de températures extrêmes des 56 stations de mesure retenues :

- Les températures minimales $(\mathrm{Tn})$, maximales $(\mathrm{Tx})$ et moyennes $(\mathrm{Tm})$ annuelles, ces dernières étant déterminées à partir de la demi-somme des températures minimales et maximales quotidiennes (Tm).

- Les écarts de températures annuels ou estivaux à la dernière normale standard 1971-2000 et à la moyenne décennale 1991-2000.

- La température maximale du jour correspondant au jour le plus chaud en moyenne sur les 56 stations disponibles.

- La température minimale correspondant à la nuit la plus chaude en moyenne sur les 56 stations disponibles.

- L'amplitude thermique diurne moyenne de la période caniculaire.

- Le nombre annuel de nuits tropicales $\left(\mathrm{Tn}>20^{\circ} \mathrm{C}\right)$.

- Le nombre annuel de jours de forte chaleur $\left(\mathrm{Tx}>30^{\circ} \mathrm{C}\right)$. représentés à l'aide de symboles ponctuels, en choisissant, à l'instar de ce qui est conventionnellement admis dans la représentation des températures, un nuancier de couleur s'étendant du bleu (signifiant froid ou plus froid que la moyenne) au rouge (signifiant chaud ou plus chaud que la moyenne) en passant par le vert et le jaune. Les valeurs d'indices de température ont été discrétisés par classe d'égale étendue. Les valeurs moyennes de $\mathrm{Tn}$, $\mathrm{Tx}$ et $\mathrm{Tm}$ ont été régionalisées par interpolation. L'interpolation de données de température ponctuelles a fait l'objet de nombreuses publications scientifiques tentant par exemple d'incorporer certaines variables physiographiques (ex. Fury et Joly, 1995). stations de mesure étant suffisamment robuste (cf. infra), celles-ci ont été spatialisées par co-krigeage simple (Wackernagel, 1998). Cette méthode est un interpolateur exact et non biaisé, qui restitue au point de mesure la valeur observée, et postule une autocorrélation spatiale de la variable d'intérêt (ici les températures) commandée partiellement par une variable auxiliaire (ici le relief). La corrélation étant nettement plus molle entre les températures minimales annuelles et l'altitude, la technique simple de la distance pondérée d'ordre 2, qui postule une décroissance parabolique du poids assigné aux stations de mesure utilisées pour déterminer la valeur en un point quelconque a été appliquée aux valeurs ponctuelles des Tn.

Elle offre l'avantage d'une spatialisation rapide de la variable d'intérêt et de l'obtention d'une surface de tendance restituant grossièrement la distribution régionale de l'information ponctuelle, le tracé des isothermes restant toutefois approximatif dans 
les régions peu documentées. Les champs de température ont été représentés sous forme de cartes isoplèthes après une discrétisation de la variable d'intérêt en classes d'égale étendue (Béguin et Pumain, 2000).

\section{Analyse des données et interprétation}

\section{A. Températures annuelles}

17 En se référant au réseau des 12 stations de mesure intangibles réparties sur le Nord-Est français et en bordure frontalière (annexe A), la température moyenne annuelle régionale de l'année 2003 s'élève à $11,4^{\circ} \mathrm{C}$ et se positionne à peu près au même niveau que l'année 2000 et l'année 1994, cette dernière étant la plus chaude des 34 dernières années. Le classement de la figure 6 indique également que l'année 2003 s'inscrit dans le sillage d'une succession d'années chaudes au cours de la décennie 1991-2000 qui totalise 8 des 10 années les plus chaudes de la normale 1971-2000 dans la région NordEst. La différence majeure entre l'année record de 1994 et 2003 réside dans l'asymétrie de l'écart des Tx à la normale $1971-2000$ (de l'ordre de 2 à $3{ }^{\circ} \mathrm{C}$ contre 1 à $1,7^{\circ} \mathrm{C}$ ) par rapport à celui des Tn en 2003, tandis que l'anomalie thermique chaude affecte aussi bien les Tn et les Tx en 1994 (tableau 1).

Figure 6 :Classement des années 1971-2000 et de l'année 2003 en fonction de leur température moyenne établie à partir des enregistrements des 12 stations de mesure intangibles du Nord-Est français.

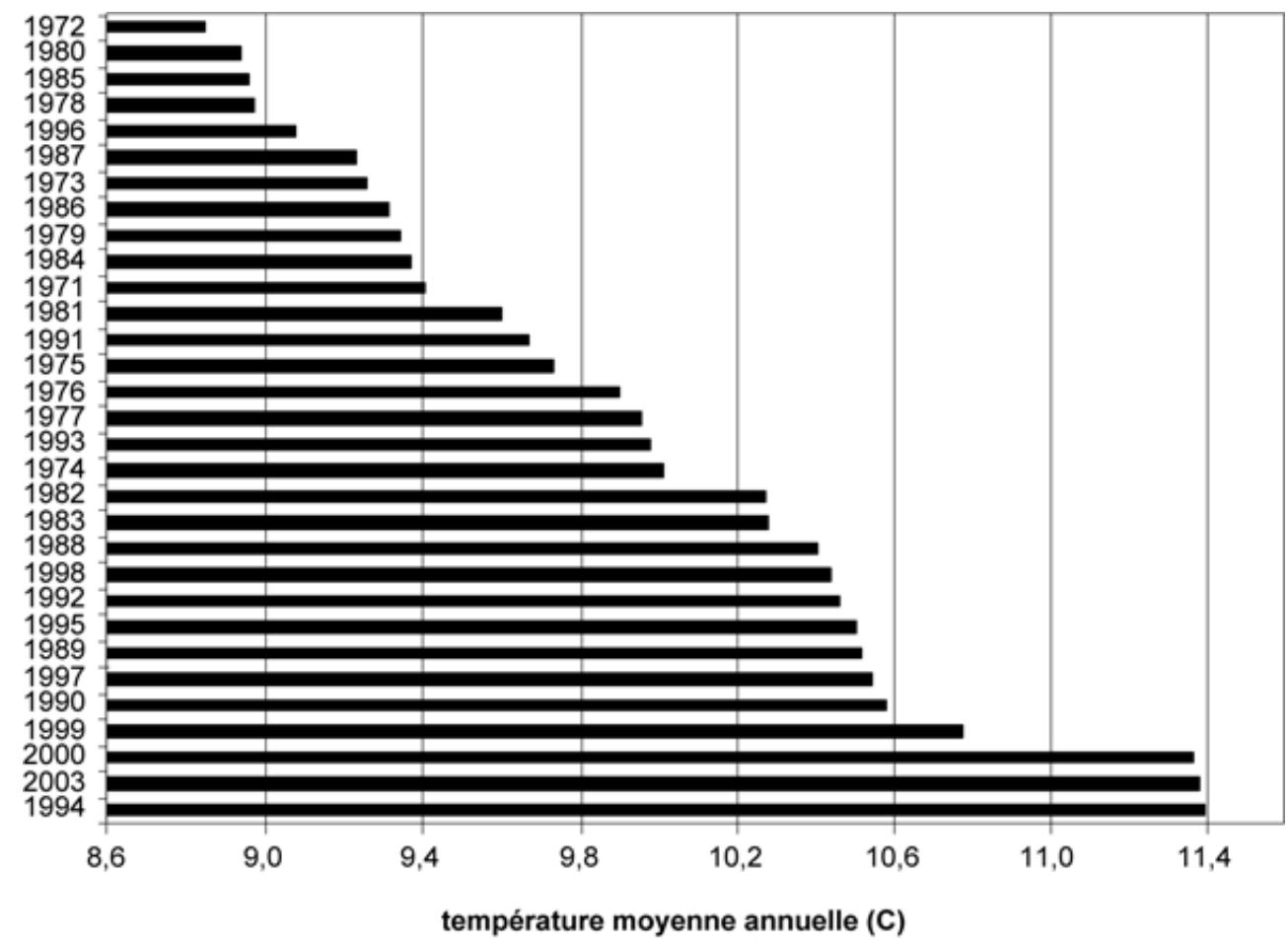


Tableau 1 : Écarts des températures minimales $(\mathrm{Tn})$, maximales $(\mathrm{Tx})$ et moyennes $(\mathrm{Tm})$ aux moyennes annuelles de la normale climatique standard 1971-2000 pour les stations de mesure intangibles du Nord-Est français.

\begin{tabular}{|c|c|c|c|c|c|c|}
\hline \multirow[t]{2}{*}{${ }^{\circ} \mathrm{C}$} & \multicolumn{3}{|c|}{1994} & \multicolumn{3}{|c|}{2003} \\
\hline & Tn & Tx & Tm & $T n$ & $T x$ & $T m$ \\
\hline AUGNY (METZ-FRESCATY) & 1,4 & 1,2 & 1,3 & 0,6 & 2,2 & 1,4 \\
\hline BELFORT & 1,7 & 1,4 & 1,6 & 0,9 & 2,3 & 1,6 \\
\hline DANNE-ET-QUATRE-VENTS (PHALSBOURG) & 1,4 & 1,2 & 1,3 & 0,6 & 2,7 & 1,7 \\
\hline ENTZHEIM (STRASBOURG-ENTZHEIM) & 1,8 & 1,2 & 1,5 & 0,5 & 2,2 & 1,3 \\
\hline ERNEVILLE-AUX-BOIS (ERNEVILLE AUX B) & 1,6 & 1,3 & 1,5 & 0,2 & 2,3 & 1,2 \\
\hline NEUFCHÂTEAU & 2,0 & 1,4 & 1,7 & 0,7 & 2,5 & 1,6 \\
\hline OCHEY (NANCY-OCHEY) & 1,5 & 1,1 & 1,3 & 0,8 & 2,2 & 1,5 \\
\hline SAINT-LOUIS (BALE-MULHOUSE) & 1,8 & 1,3 & 1,5 & $-0,1$ & 2,1 & 1,0 \\
\hline SAINT-SAUVEUR (LUXEUIL) & 2,2 & 1,2 & 1,7 & 0,7 & 2,1 & 1,4 \\
\hline SEWEN (SEWEN - LAC) & 1,8 & 1,7 & 1,7 & 1,7 & 3,0 & 2,3 \\
\hline STRASBOURG (STG - BOT. SA) & 1,4 & 1,0 & 1,2 & 0,2 & 2,1 & 1,2 \\
\hline TOMBLAINE (NANCY-ESSEY) & 1,7 & 1,3 & 1,5 & 0,5 & 2,3 & 1,4 \\
\hline MOYENNE & 1,7 & $\mid 1,3$ & 1,5 & 0,6 & 2,3 & 1,5 \\
\hline
\end{tabular}

On notera également que l'écart à la normale des Tx est assez homogène sur le plan régional, la plus forte amplitude affectant la station de moyenne montagne de SewenLac située dans les Hautes Vosges. La répartition géographique des températures maximales moyennes annuelles (figure 7a) laisse apparaître sans surprise un déterminisme altitudinal, les valeurs de Tx suivant un gradient thermique vertical de l'ordre de $-0,6^{\circ} \mathrm{C} / 100 \mathrm{~m}\left(\mathrm{Tx}=-0,0062 \mathrm{Z}+18,3, \mathrm{R}^{2}=0,78\right)$, le maximum régional étant enregistré à la station bas-rhinoise de Stattmatten $\left(18,1^{\circ} \mathrm{C}\right)$, contre un minimum à la station des Hautes-Vosges du Markstein avec 10,4 ${ }^{\circ} \mathrm{C}$. En 2003, les nuits les plus fraîches ont été observées au Markstein $\left(3,7^{\circ} \mathrm{C}\right)$ et les plus chaudes dans le jardin botanique de Strasbourg $\left(7,7^{\circ} \mathrm{C}\right)$ (figure $\left.7 \mathrm{~b}\right)$. À altitude équivalente, l'année 2003 aura été marquée par des nuits plus fraîches dans l'Argonne (ex. Erneville-aux-bois), en Barrois (ex. Maxey), dans le Xantois (ex. Neufchâteau), ou encore dans le pays de Bitche (ex. Moutherhouse), par rapport au piedmont vosgien (ex. Bergheim) (figure 7b). 
Figure 7a :Distribution régionale des températures maximales moyennes annuelles pour 2003.

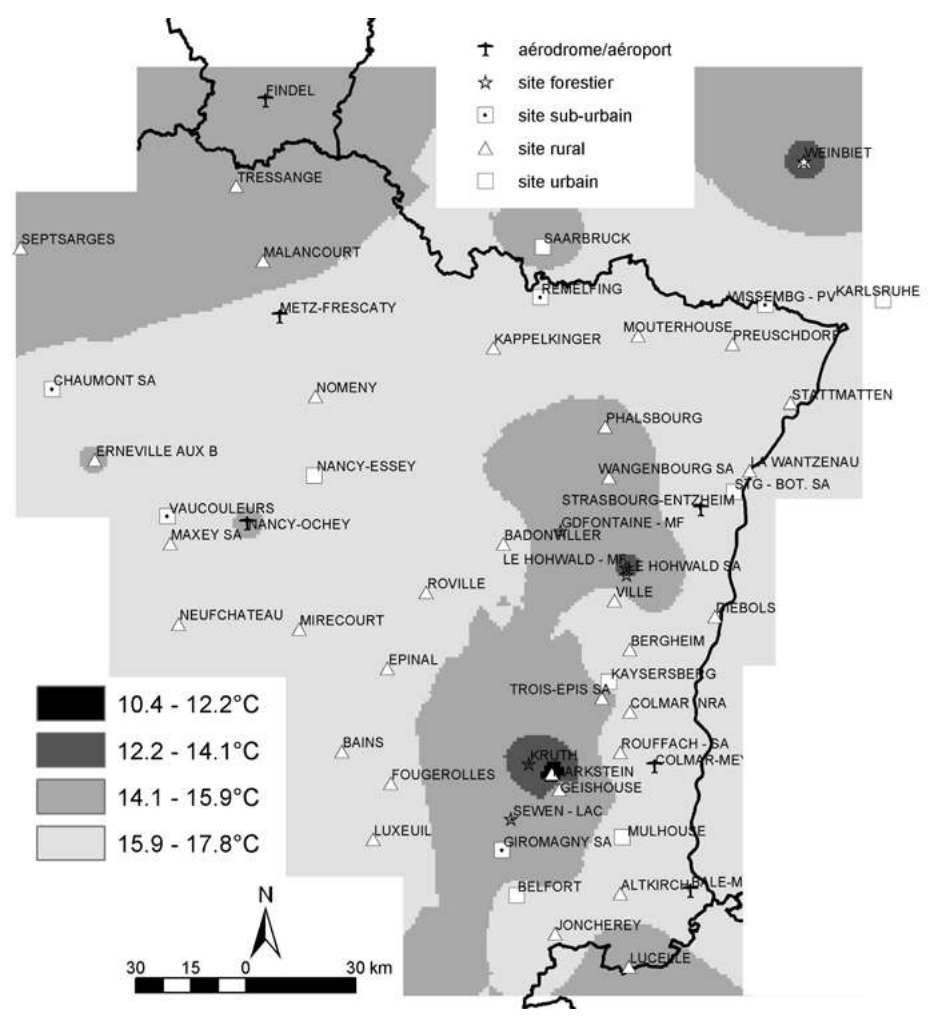

Figure $7 \mathrm{~b}$ : Distribution régionale des températures minimales moyennes annuelles pour 2003.

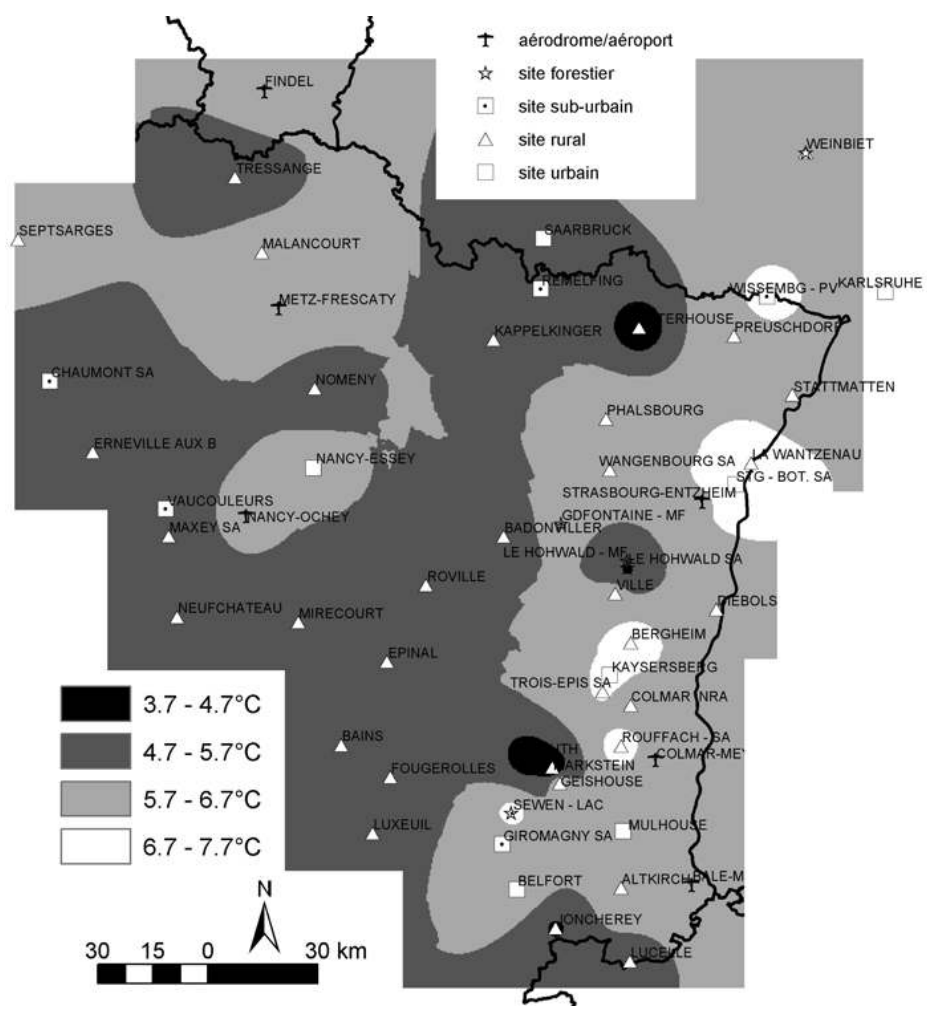


Figure 7c : Distribution régionale des températures moyennes annuelles pour 2003.

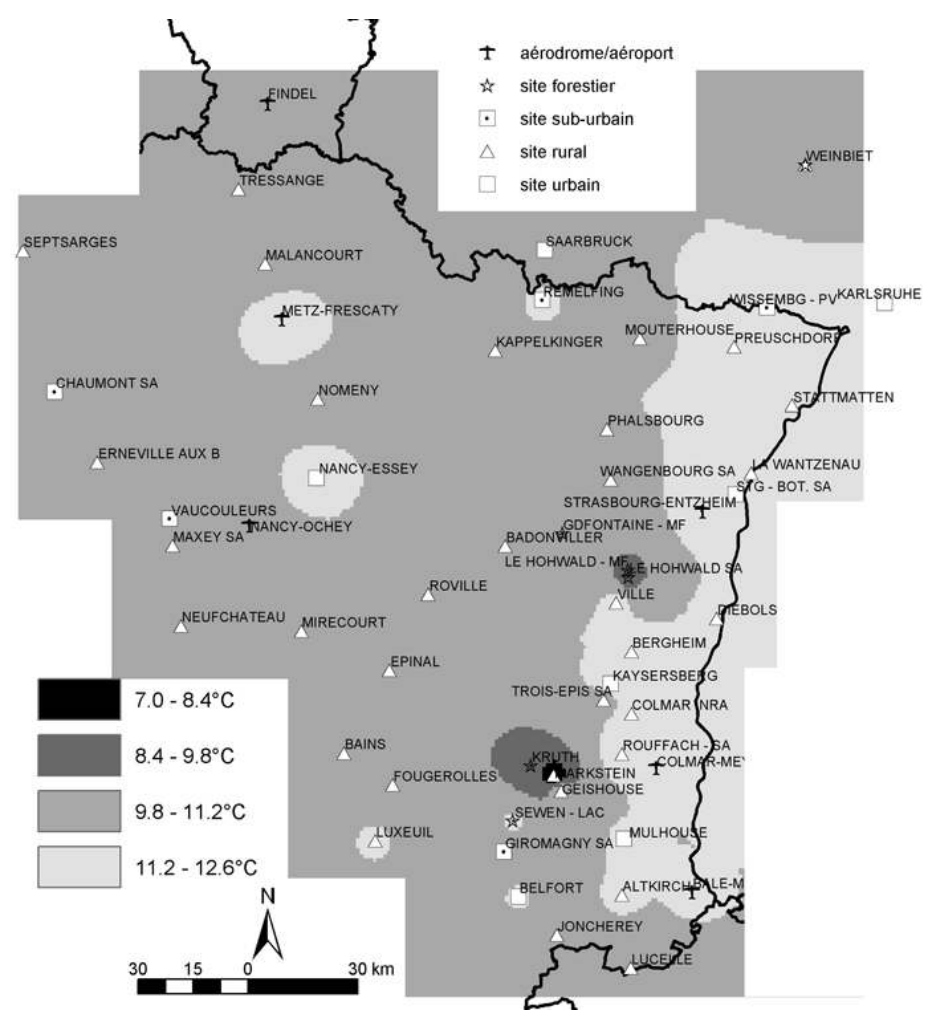

19 La relation entre les valeurs annuelles des Tn et l'altitude des stations de mesure est beaucoup plus dispersée compte tenu des multiples effets de site qui entrent en jeu et qui sont susceptibles, malgré une décroissance régionale des températures moyennes annuelles selon l'altitude (figure $7 \mathrm{c}$ ), d'inverser localement le gradient thermique vertical. L'écart entre les températures moyennes $\left(-0,5^{\circ} \mathrm{C}\right)$ des stations de Maxey SA $\left(250 \mathrm{~m}, 10,7^{\circ} \mathrm{C}\right)$ et Vaucouleurs $\left(294 \mathrm{~m}, 11,2^{\circ} \mathrm{C}\right)$ situées toutes deux au pied du front de la côte des Bars est éloquent à cet égard. Le site sub-urbain occupé par la station de Vaucouleurs (figure 8a) se traduit par des nuits plus douces à la faveur probablement d'un réchauffement plus important des masses d'air par la base au contact de surfaces minérales plus chaudes en journée par rapport à la station de Maxey (figure 8b), dont l'environnement s'échauffe moins en journée et qui par son caractère rural est moins influencé par l'effet de chaleur urbain nocturne. La même hiérarchie des valeurs extrêmes de $\mathrm{Tn}$ se retrouve pour la température moyenne annuelle régionale (figure 7c), puisque la station de Strasbourg-ville (STG-BOT SA) enregistre la Tm la plus douce $\left(12,6^{\circ} \mathrm{C}\right)$ et la station du Markstein la plus basse $\left(7^{\circ} \mathrm{C}\right)$. 
Figure 8a :Localisation de la station de mesure meusienne de Vaucouleurs.

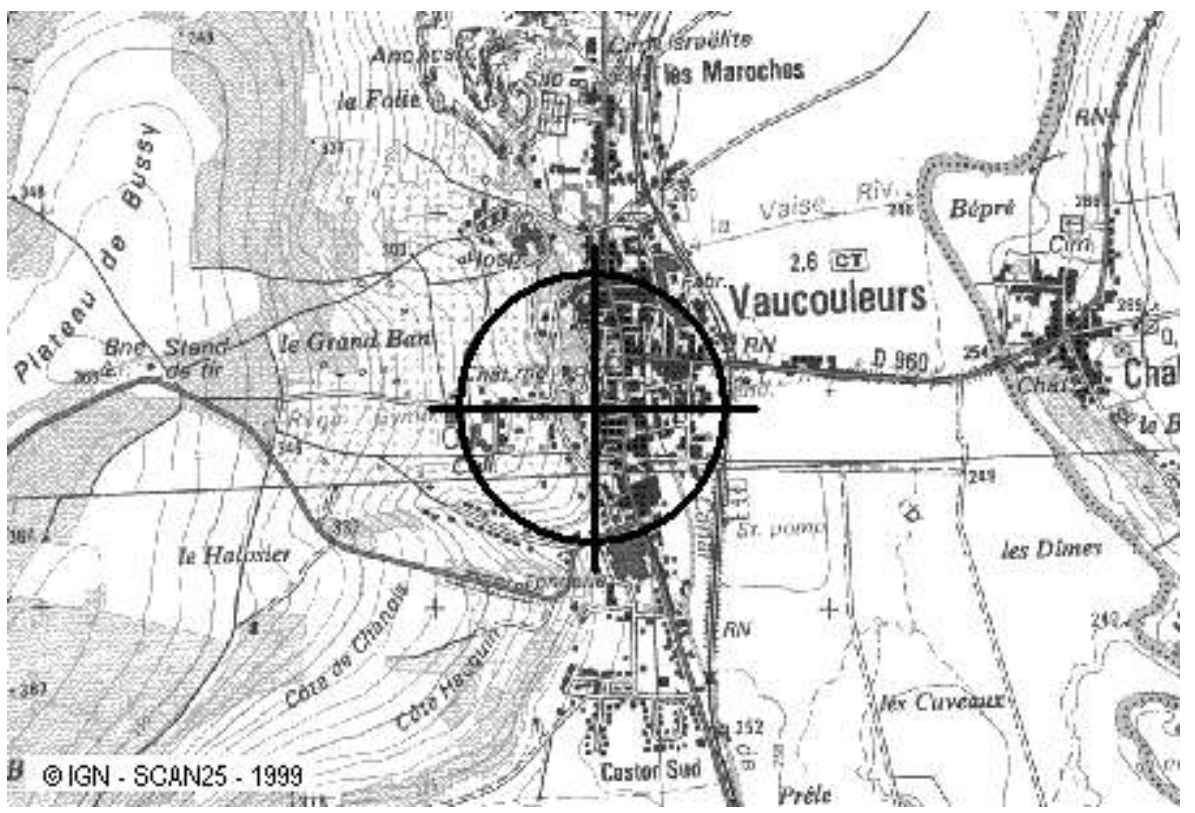

D'après les renseignements fournis par le service Climathèque de Météo-France.

Figure $8 b$ :Localisation de la station de mesure meusienne de Maxey SA (B).

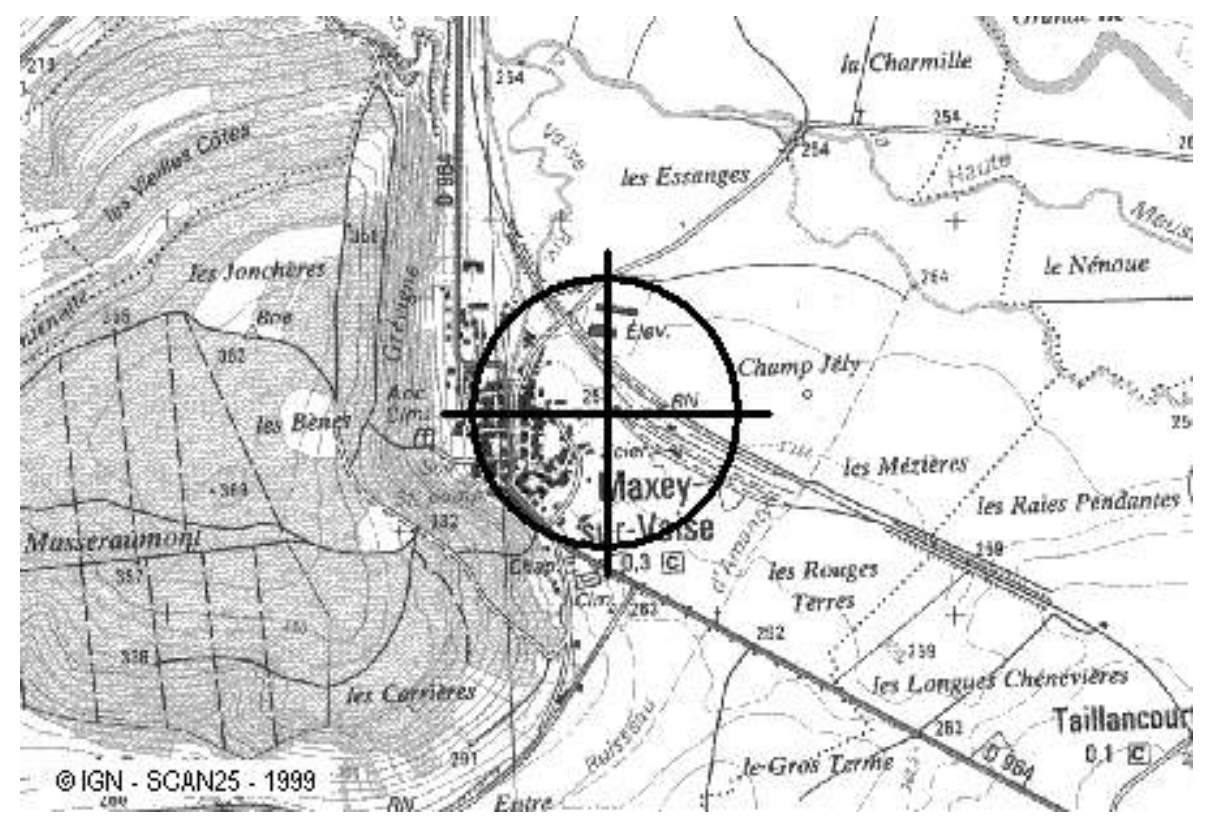

D’après les renseignements fournis par le service Climathèque de Météo-France.

Les écarts de température des Tn à la moyenne décennale 1991-2000 opposent (figure 9a) :

- D'une part des stations enregistrant des Tn légèrement plus fraîches que la moyenne décennale, jusqu'à $-0,5{ }^{\circ} \mathrm{C}$, et occupant des zones topographiquement déprimées : fond de vallées incisant le plateau lorrain (ex. Kappelkinger), plaine d'Alsace (ex. Diebolsheim), site forestier à fond plat (ex. Mouterhouse) plus affectés par la stagnation d'air froid produit par rayonnement nocturne. 
- D'autre part des stations de revers de côte (ex. Malancourt) de moyenne montagne (ex. Sewen-Lac) ou de colline sous-vosgienne (ex. Bergheim) marquées par des Tn plus douces que sur la décennie 1991-2000.

Figure 9a : Écarts des températures minimales de 2003 aux moyennes décennales de la décennie 1991-2000 pour les stations de mesure intangibles du Nord-Est français et ses bordures luxembourgeoise et allemande.

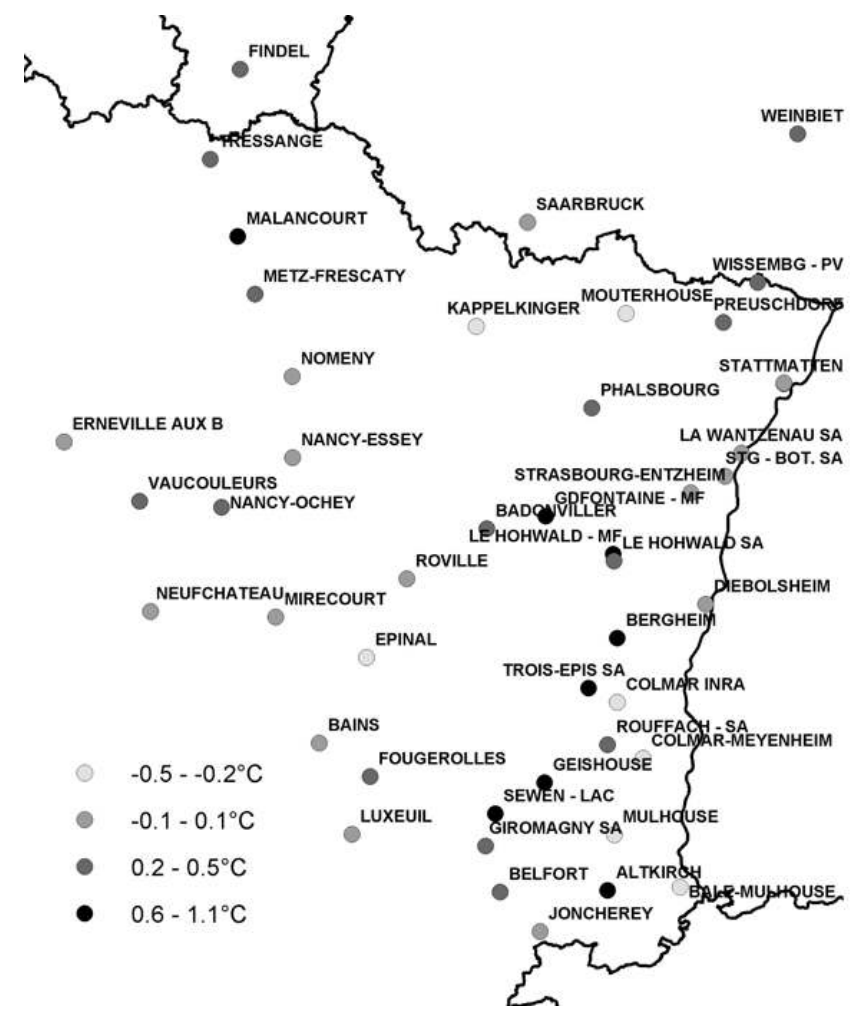


Figure $9 \mathrm{~b}$ :Écarts des températures maximales de 2003 aux moyennes décennales de la décennie 1991-2000 pour les stations de mesure intangibles du Nord-Est français et ses bordures luxembourgeoise et allemande.

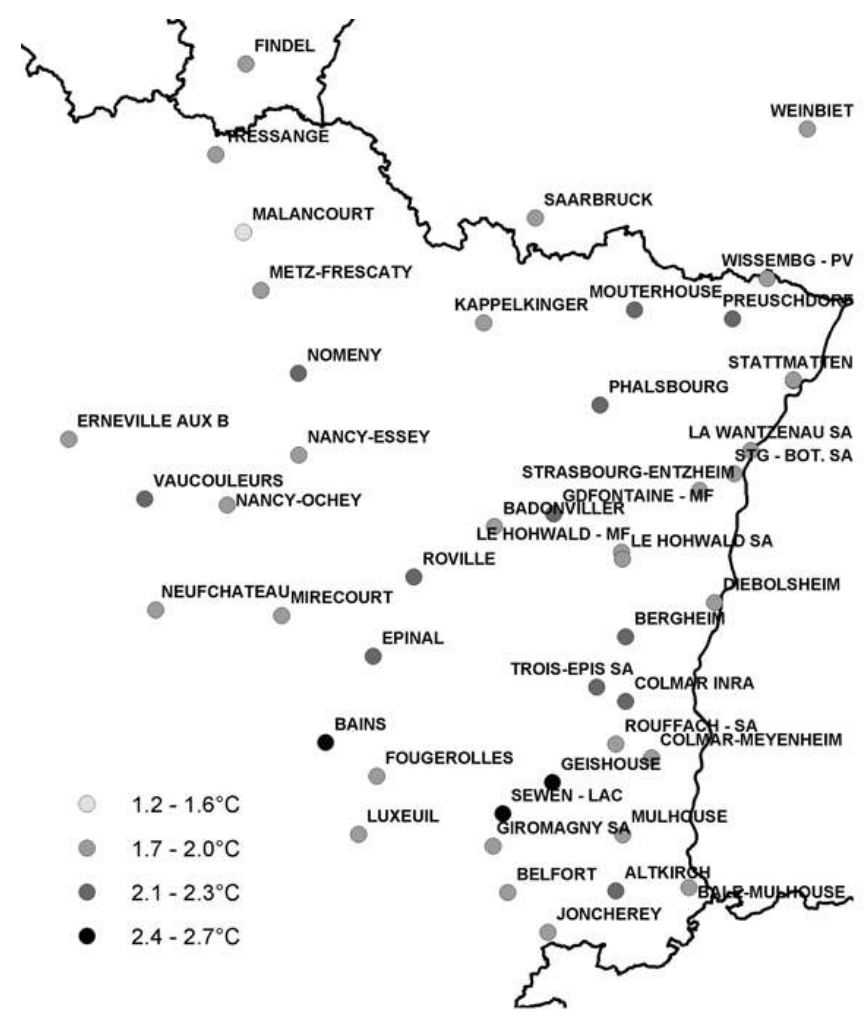

La persistance en 2003, d'un régime anticyclonique et la fréquence bien supérieure à la moyenne décennale de nuits claires et peu nuageuses, propices à la formation fréquente d'inversions thermiques nocturnes dès l'amorce du printemps, est sans aucun doute à l'origine de cette différenciation régionale. La distribution spatiale des anomalies chaudes des valeurs annuelles de $\mathrm{Tx}$ (figure $9 \mathrm{~b}$ ), montre l'existence d'écarts à la moyenne décennale moins prononcés $\left(<2^{\circ} \mathrm{C}\right)$ pour les stations de mesure situées en fond de vallée (ex. Nancy-Essey), en plaine (ex. Metz-Frescaty) ou dans une vallée intramontagnarde où l'impact d'un masque topographique gomme l'influence d'une situation d'adret (ex. Trois-épis SA).

Les valeurs d'anomalies thermiques supérieures à $+2,2{ }^{\circ} \mathrm{C}$ concernent une large gamme de sites topoclimatiques : on notera dans ce lot la présence de stations d'adret rurales (ex. Preuschdorf), de stations de moyenne altitude en sommet de pente (ex. Altkirch) ou de stations d'adret forestières (ex. Mouterhouse).

\section{A. Caractéristiques thermiques de la saison estivale 2003}

\section{Aspects généraux}

L'examen cursif de la répartition régionale des écarts de température des nuits du mois de juin (figure 10a) par rapport à la décennie chaude 1991-2000 révèle deux tendances nettes : d'une part les écarts sont généralement plus creusés en plaine d'Alsace et en Haute-Saône $\left(>+4^{\circ} \mathrm{C}\right)$ par opposition au plateau lorrain $\left(2\right.$ à $\left.4{ }^{\circ} \mathrm{C}\right)$ et d'autre part un étagement altitudinal se dessine, les stations situées au-dessus de $500 \mathrm{~m}$ d'altitude (Altkirch, Geishouse, Le Hohwald M-F, Lucelle, Sewen-Lac et Trois-Épis) étant celles qui 
enregistrent les plus grands écarts de température par rapport à la moyenne décennale (record pour Sewen-Lac avec $+5,5^{\circ} \mathrm{C}$ ). L'étagement altitudinal des anomalies thermiques des Tn est valable au mois de juillet (figure 10b) et $a$ fortiori au mois d'août (figure 10c) où sur les 14 stations de mesure enregistrant un écart à la moyenne décennale supérieur à $3{ }^{\circ} \mathrm{C}$ (record pour Weinbiet avec $+4,4{ }^{\circ} \mathrm{C}$ ), 12 sont situées à plus de 350 m d'altitude.

Figure 10a :Écarts des températures minimales de juin 2003 aux moyennes décennales de la décennie 1991-2000 pour les stations de mesure intangibles du Nord-Est français et ses bordures luxembourgeoise et allemande.

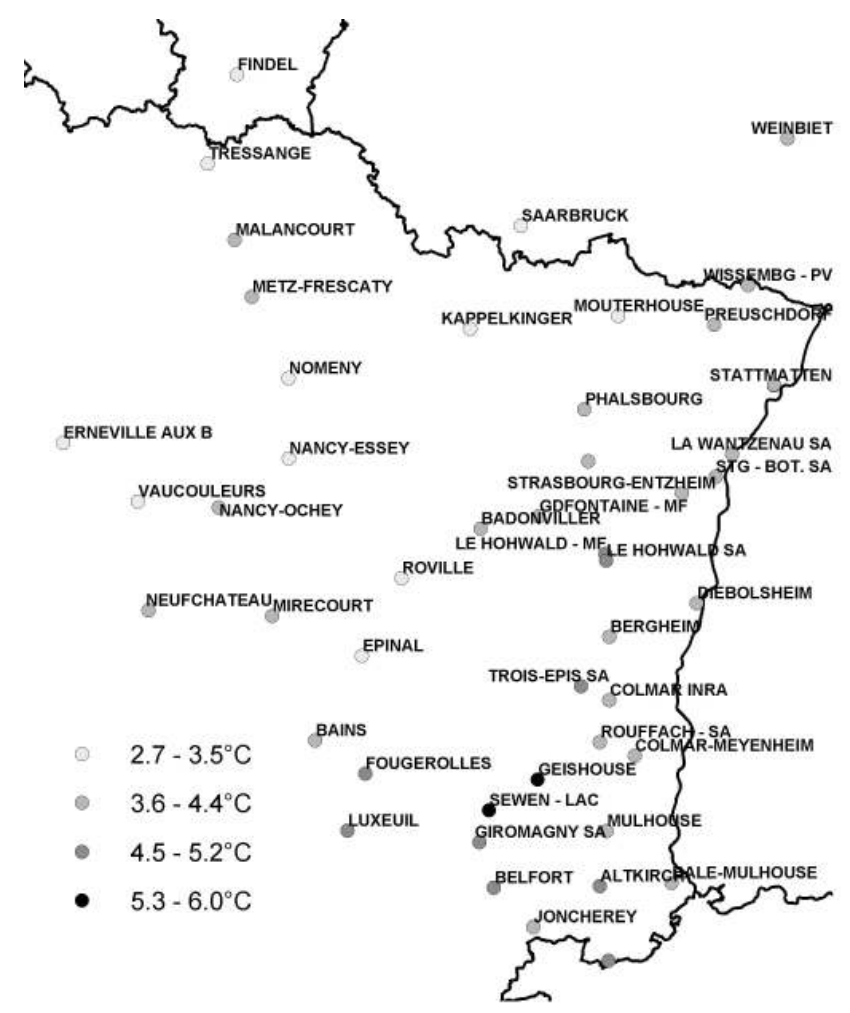


Figure 10b :Écarts des températures minimales de juillet 2003 aux moyennes décennales de la décennie 1991-2000 pour les stations de mesure intangibles du Nord-Est français et ses bordures luxembourgeoise et allemande.

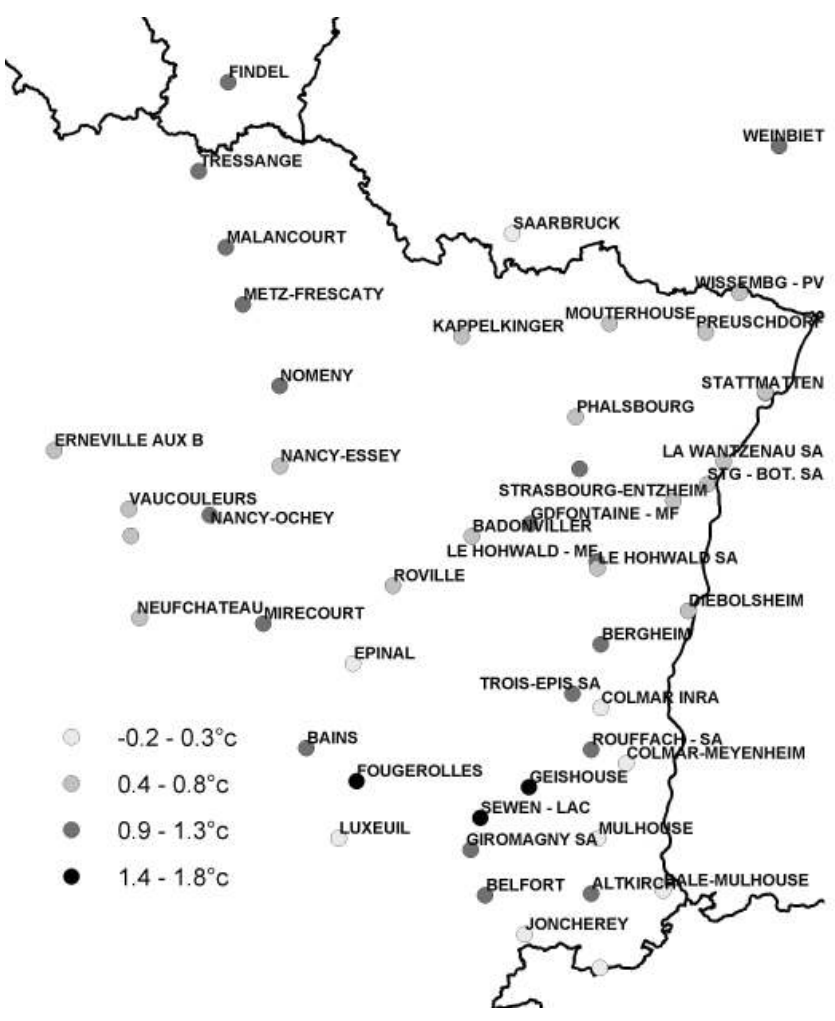

Figure 10c :Écarts des températures minimales d'août 2003 aux moyennes décennales de la décennie 1991-2000 pour les stations de mesure intangibles du Nord-Est français et ses bordures luxembourgeoise et allemande.

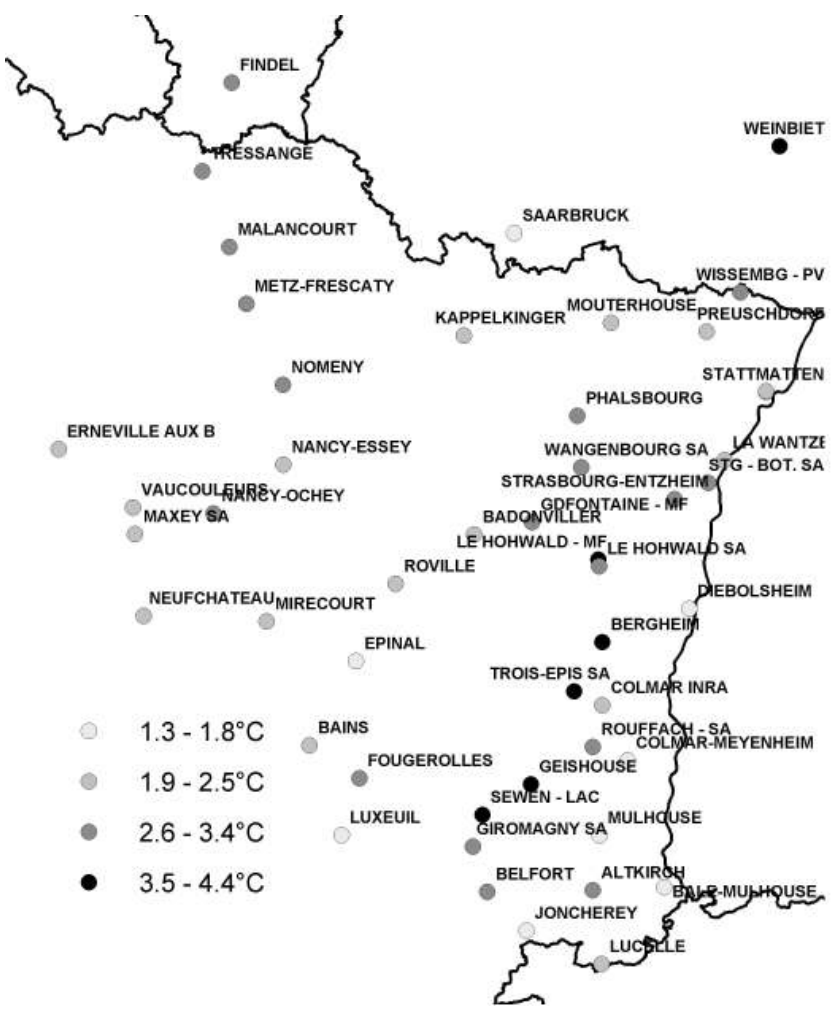


Les températures extrêmes de l'année 2003 dans le Nord-Est français et ses bo...

19

Figure 10d :Écarts des températures maximales de juin 2003 aux moyennes décennales de la décennie 1991-2000 pour les stations de mesure intangibles du Nord-Est français et ses bordures luxembourgeoise et allemande.

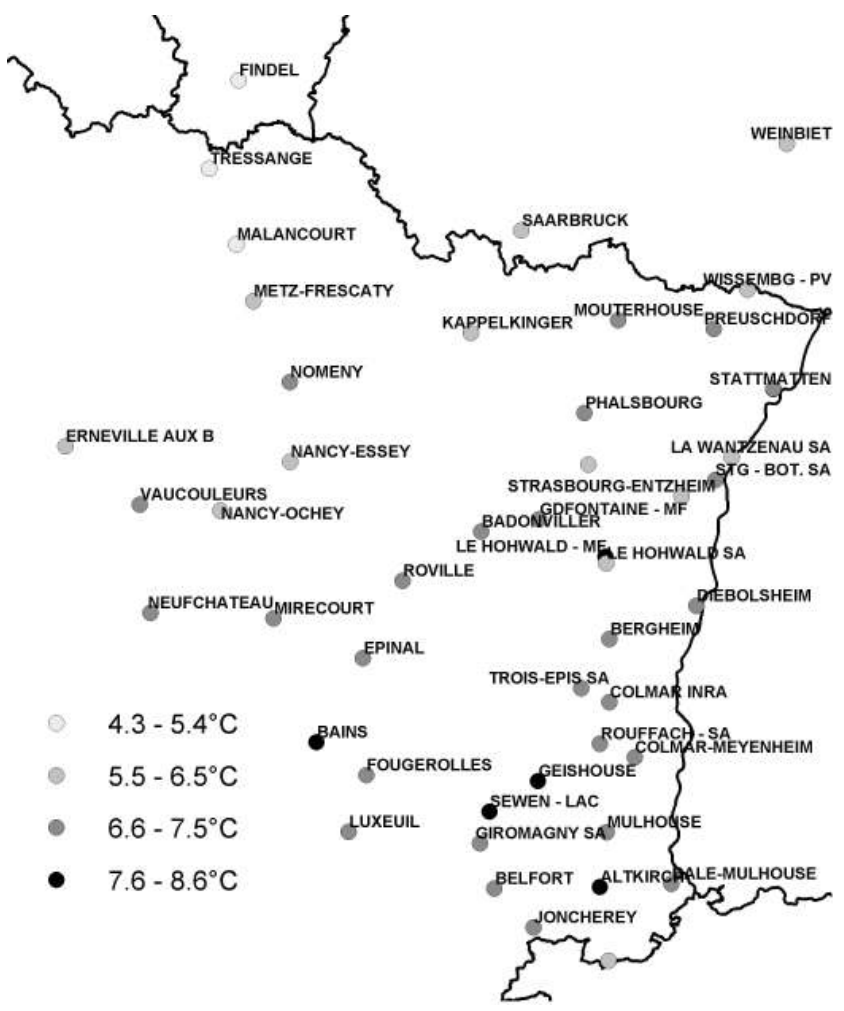

Revue Géographique de l'Est, vol. 45 / 2 | 2005 
Figure 10e :Écarts des températures maximales de juillet 2003 aux moyennes décennales de la décennie 1991-2000 pour les stations de mesure intangibles du Nord-Est français et ses bordures luxembourgeoise et allemande.

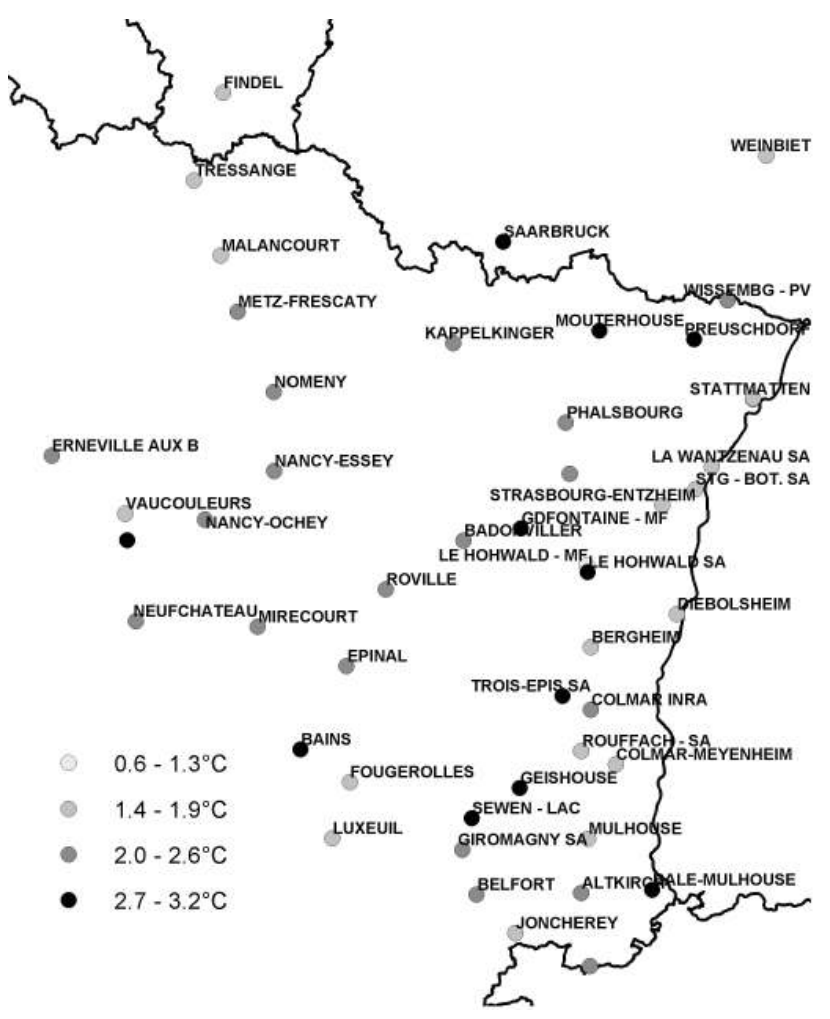

Figure $10 f$ :Écarts des températures maximales d'août 2003 aux moyennes décennales de la décennie 1991-2000 pour les stations de mesure intangibles du Nord-Est français et ses bordures luxembourgeoise et allemande.

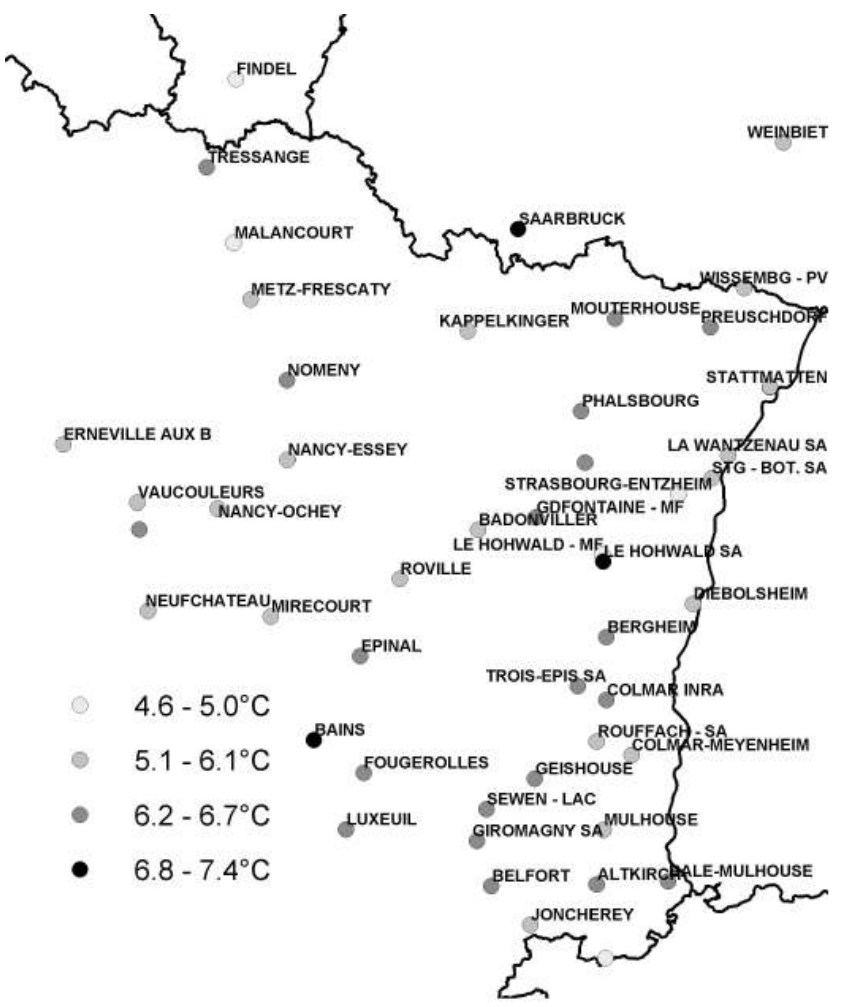


Les nuages de points des figures $11 \mathrm{a}$ et $11 \mathrm{~b}$ confirment l'existence d'une dépendance altitudinale des anomalies de température au-delà du seuil de $500 \mathrm{~m}$, fait relevé par ailleurs par Beniston et Rebetez (1996) sur les anomalies chaudes des Tn en conditions anticycloniques hivernales en Suisse, cette dépendance augmentant linéairement avec l'altitude. Cette linéarité est plus prononcée au mois d'août qu'au mois de juin sans doute en raison de la plus grande homogénéité des anomalies de Tx au-delà de $500 \mathrm{~m}$ en août qu'en juin.

La disparité des écarts de température à la moyenne décennale est légèrement plus forte au mois d'août à basse altitude. Les écarts à la moyenne décennale des Tx de juin, juillet et août (figures $10 \mathrm{~d}, 10 \mathrm{e}$ et 10f) sont davantage commandés par l'apport énergétique diurne d'origine advective et radiative et donc également l'orientation du versant qui caractérise chaque station de mesure. Les anomalies du mois de juin sont légèrement supérieures à celles du mois d'août (figure $10 \mathrm{~d}$ versus 10f), les premières étant causées par un ensoleillement exceptionnel, les secondes par un afflux généralisé d'air chaud d'origine tropicale dans la basse et la moyenne troposphère, aggravé par une insolation élevée.

Figure 11a :Évolution des anomalies de température minimale à la décennie 1991-2000 au mois de juin 2003 en fonction de l'altitude de la station de mesure.

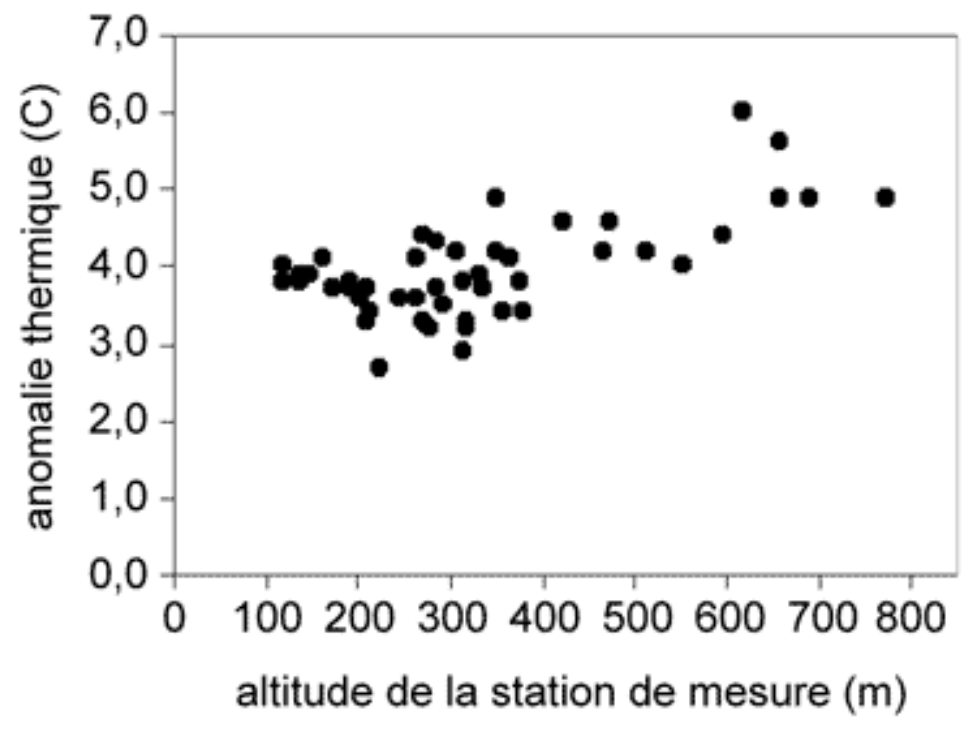


Figure 11b : Évolution des anomalies de température minimale à la décennie 1991-2000 au mois d'août 2003 en fonction de l'altitude de la station de mesure.

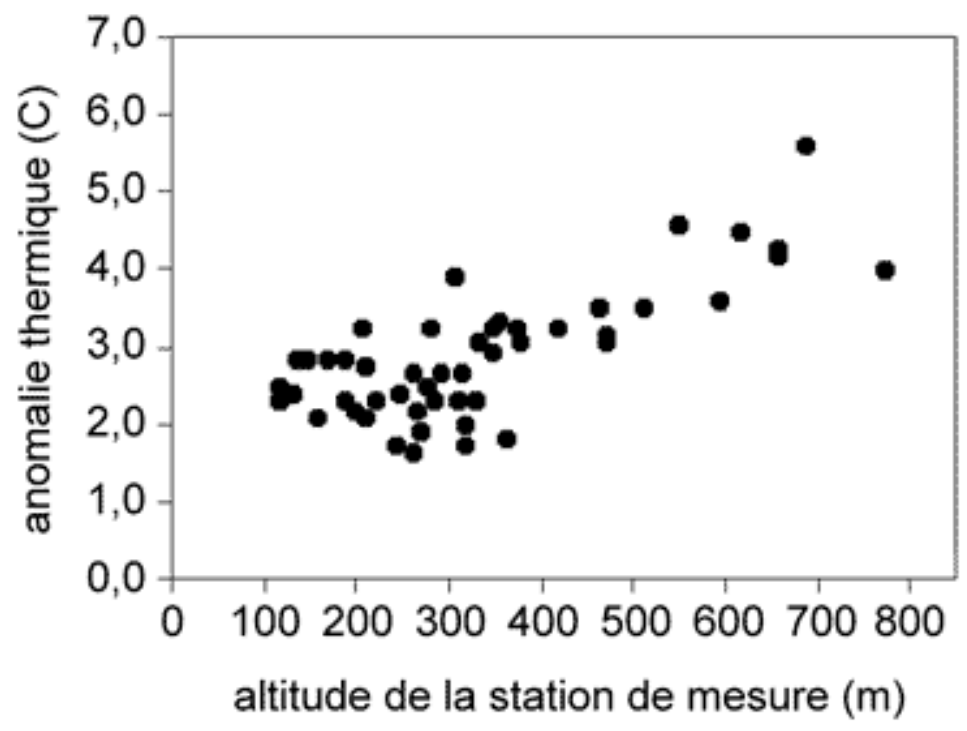

Les plus grands écarts de température du mois de juin sont enregistrés pour des stations situées sur le pourtour ou au sein du massif vosgien (maximum pour Geishouse dans les Hautes-Vosges avec $+8,6^{\circ} \mathrm{C}$ ), tandis que les écarts sont plus ténus en plaine d'Alsace et sur le plateau lorrain. Pour la station de Lucelle située à $690 \mathrm{~m}$ d'altitude sur les premiers contreforts jurassiens (figure 12), il est probable que la présence d'un masque orographique vers le sud (chaînons du Jura) maintenant le site à l'ombre plus longtemps le matin, et la formation de brises thermiques assurant une certaine fraîcheur le jour en sommet de pente, puissent expliquer la faible anomalie de température observée en juin et plus encore au mois d'août (figure 10f).

Figure 12 :Localisation de la station de mesure haut-rhinoise de Lucelle.

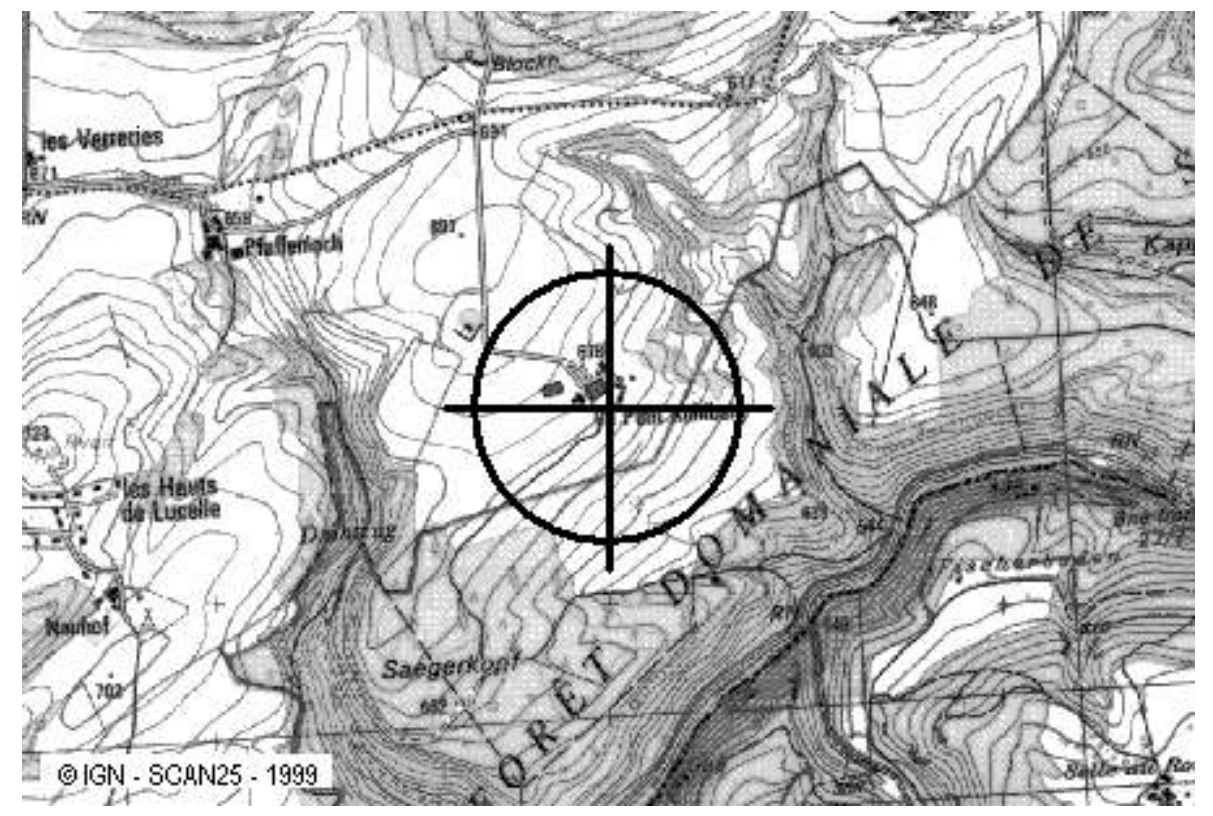

D’après les renseignements fournis par le service Climathèque de Météo-France. 


\section{La vague de chaleur du mois d'août}

Elle débute dans le Nord-Est français le 2 août, premier jour de forte chaleur $\left(\mathrm{Tx}>30^{\circ} \mathrm{C}\right.$ en moyenne régionale) pour s'achever le 14 août, dernier jour de forte chaleur. Cette séquence de 13 jours consécutifs de forte chaleur est exceptionnelle. La cartographie des Tx enregistrées le 12 août 2003 (figure 13), date d'occurrence du jour le plus chaud en moyenne sur les 56 stations disponibles, fait apparaître un chapelet de stations très chaudes dans la vallée de la Meuse (Chaumont SA, Maxey SA, etc.), de la Meurthe (ex. Nancy Essey) et de la Moselle (ex. Metz-Frescaty).

Figure 13 :Distribution régionale des températures maximales enregistrées le 12 août 2003.

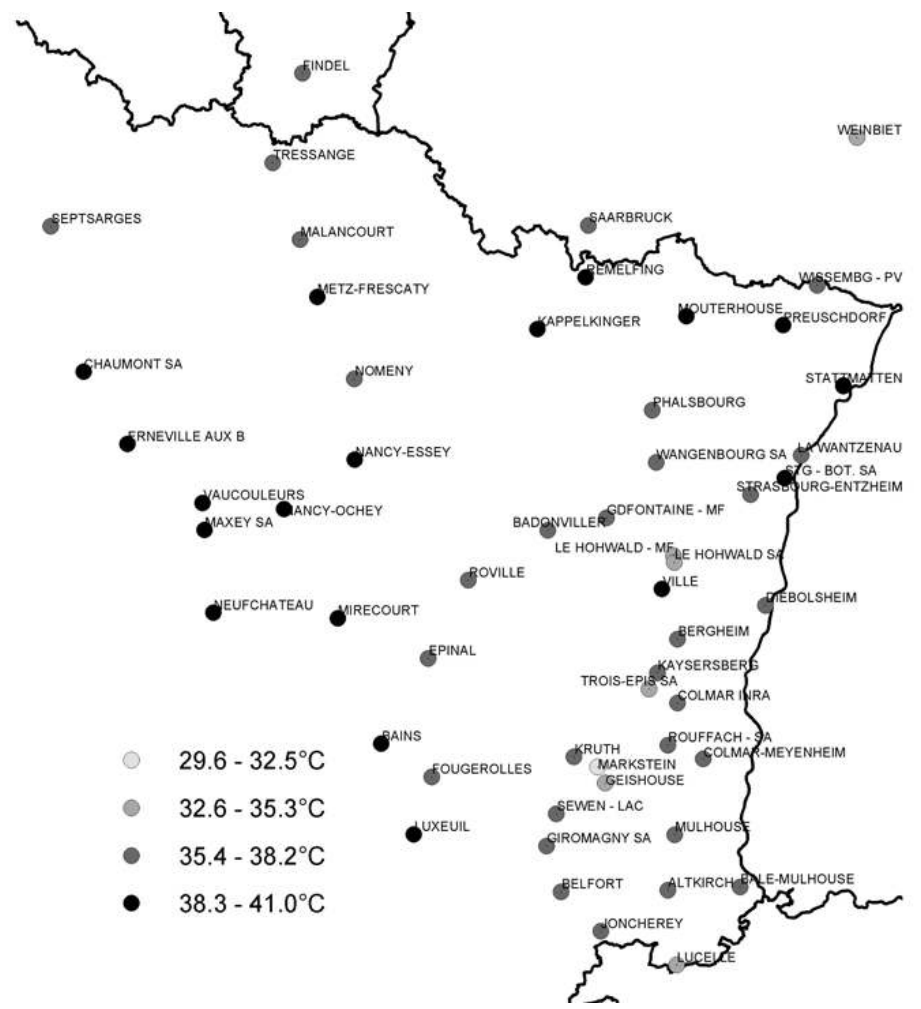

Le 12 août 2003 , la Tx la plus faible est enregistrée au Markstein, $29,6^{\circ} \mathrm{C}$, ce qui repousse très loin en altitude, vers $4600 \mathrm{~m}$, l'isotherme $0^{\circ} \mathrm{C}$. Bien que l'altitude soit le premier facteur explicatif de la variabilité régionale des Tx absolues du 12 août 2003 (figure 14) différents facteurs contribuent à aggraver localement l'extrême chaleur de cette journée :

- Une situation de fond de vallée étroite et profonde propice au confinement de l'air chaud dans les basses couches de l'atmosphère. Ainsi, la station de Sewen-Lac (620 m fond de vallée) située dans une ancienne vallée glaciaire enregistre une Tx de $36,5{ }^{\circ} \mathrm{C}$ contre $34,8{ }^{\circ} \mathrm{C}$ pour la station de Geishouse $(660 \mathrm{~m})$ exposée plein sud sur un versant dégagé et mieux ventilé : le gradient thermique vertical moyen de $-0,8^{\circ} \mathrm{C}$ dérivé du nuage de points de la figure 14 ne suffit pas à expliquer la différence de $1,7^{\circ} \mathrm{C}$ entre les deux stations.

- L'influence du bâti urbain générant des écarts ville-campagne importants. Ils sont par exemple de l'ordre de $2,2{ }^{\circ} \mathrm{C}$ entre Strasbourg-ville (139 m) et La Wantzenau (136 m).

- Des conditions de ventilation déficiente propres à l'effet de cuvette confinée et à proximité d'une agglomération de taille moyenne, comme dans le cas de la station de mesure de Bains. 
Figure 14 : Évolution des températures maximales enregistrées le 12 août 2003 en fonction de l'altitude de la station de mesure.

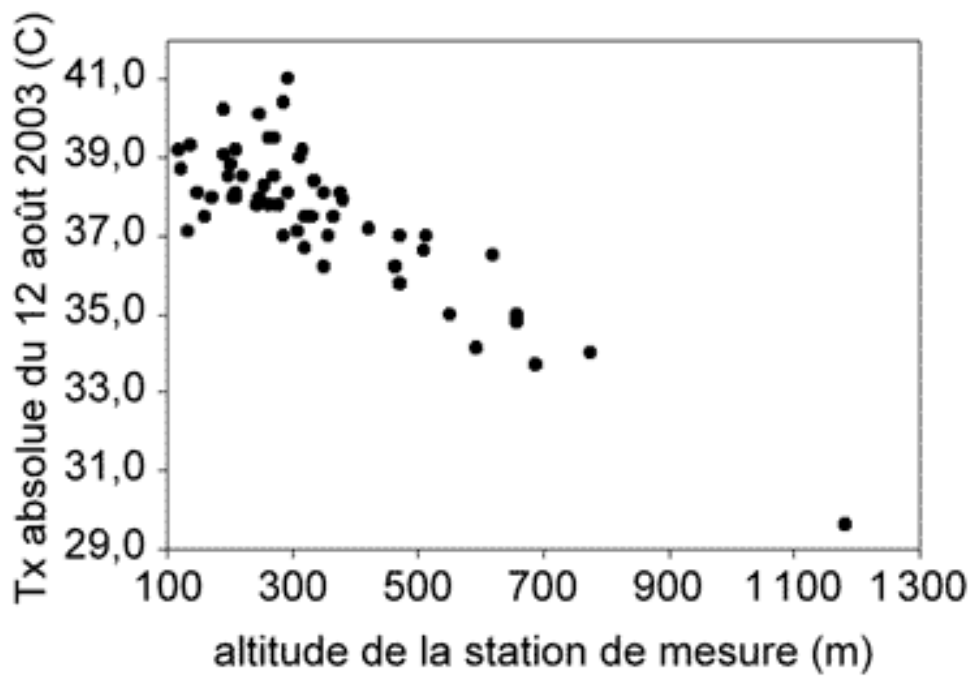

En 2003, le record absolu est détenu par Vaucouleurs avec une Tx de $41,0^{\circ} \mathrm{C}$ le 12 août ; au total 5 stations de mesure ont enregistré une valeur de Tx excédant $40^{\circ} \mathrm{C}$ (ColmarMeyenheim, Maxey SA, Neufchâteau et Preuschdorf). Concernant la distribution régionale des températures minimales coïncidant avec la nuit la plus douce sur les 56 stations disponibles (figure 15), il est intéressant de constater que les stations de mesure situées en moyenne montagne, au-delà de $500 \mathrm{~m}$ d'altitude (Altkirch, Geishouse, Le Hohwald M-F, Sewen-Lac, Trois-Épis, Weinbiet) ont connu une nuit aussi chaude sinon plus chaude que les stations de mesure d'altitude inférieure, aucun gradient thermique vertical ne se dessinant clairement (figure 16). 
Figure 15 :Distribution régionale des températures minimales enregistrées le 06 août 2003.

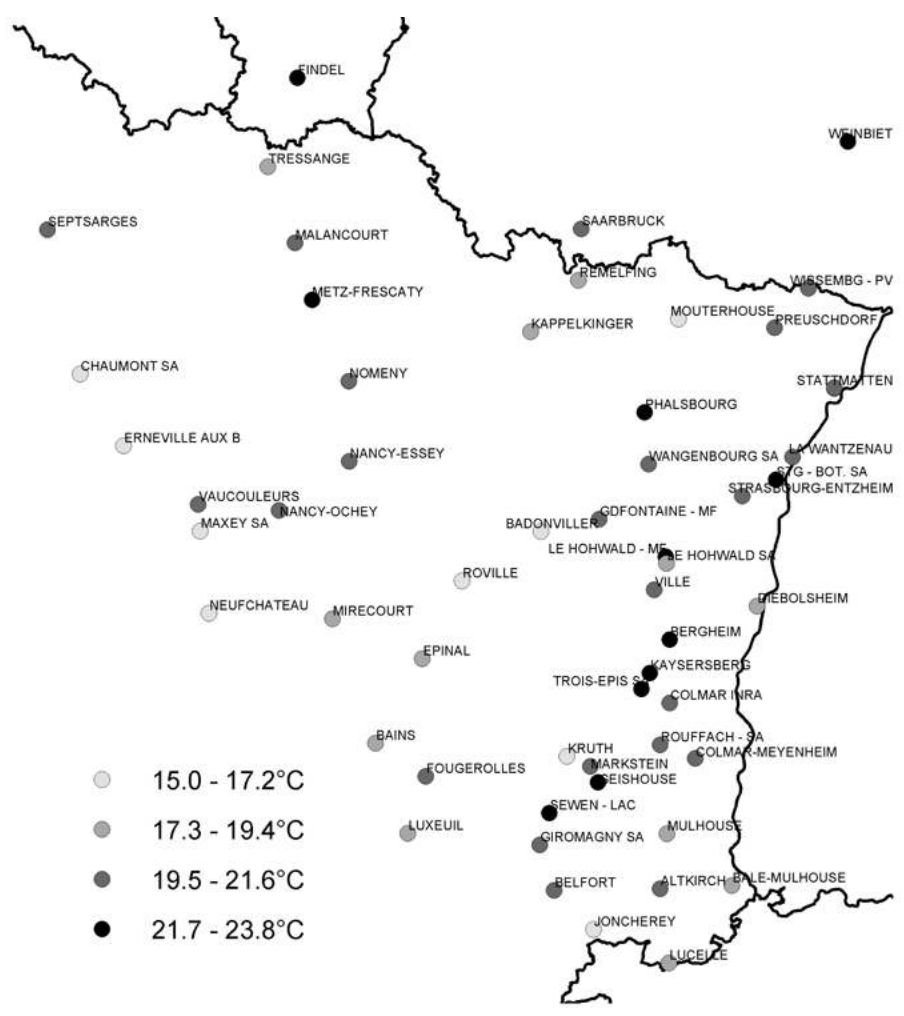

Figure 16 : Évolution des températures minimales enregistrées le 06 août 2003 en fonction de l'altitude de la station de mesure.

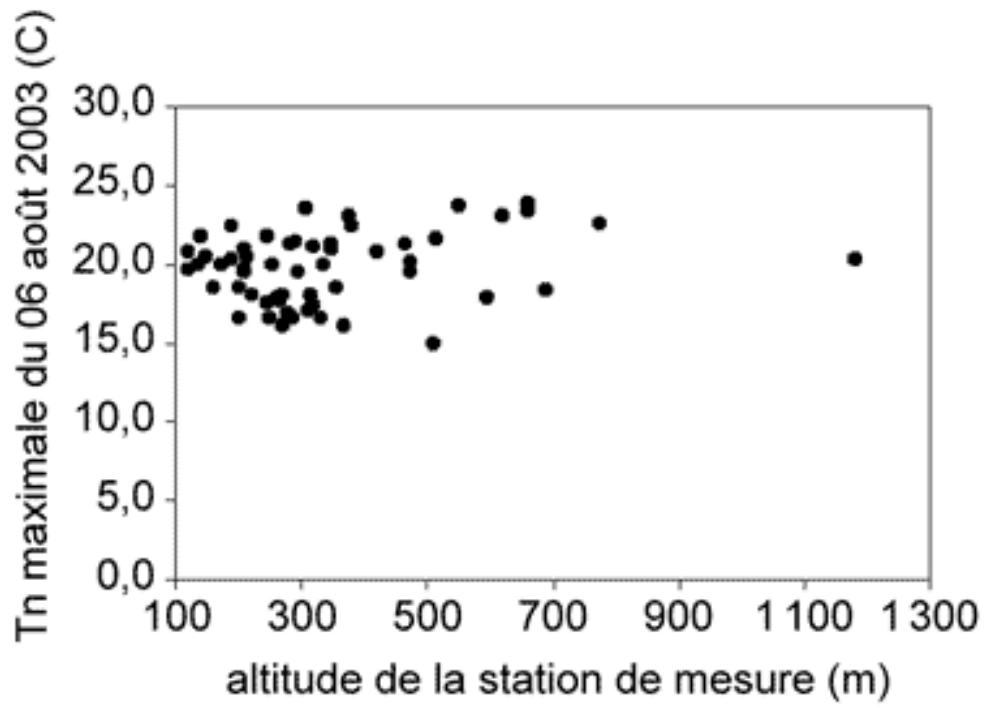

La nuit du 06 août, la température minimale la plus «torride » a été enregistrée à la station de Turckheim (Trois-Épis SA) avec $23,8^{\circ} \mathrm{C}$ à $660 \mathrm{~m}$ d'altitude. Sur la crête des Hautes-Vosges (station du Markstein), la température de l'air était encore de $20,2^{\circ} \mathrm{C}$ avant le lever du soleil le 06 au matin soit à peine $0,1^{\circ} \mathrm{C}$ de plus que la Tn de Giromagny située au pied des Hautes-Vosges à $473 \mathrm{~m}$ d'altitude. En termes de températures potentielles, il fait donc plus chaud la nuit sur la crête vosgienne qu'à basse altitude. 
Selon toute vraisemblance, deux facteurs se conjuguent pour expliquer l'occurrence de ce gradient de températures potentielles positif :

- des écoulements d'air liés aux brises de montagne qui favorisent l'afflux d'air frais dans les basses couches en vallée ou en plaine et une advection d'air chaud dans l'atmosphère libre, comme a pu le montrer Kastendeuch (1996) dans les Hautes Vosges au cours de l'été 1994;

- l'épaisseur atmosphérique touchée par l'afflux d'air chaud pendant la période caniculaire (entre 4000 et $5000 \mathrm{~m}$ ), qui a engendré des Tx très élevées à toute altitude. Pour mieux mettre en perspective ces constatations, l'amplitude thermique diurne (ATD) journalière moyenne mesurée au cours de la quinzaine caniculaire du mois d'août a été déterminée pour les 56 stations de mesure disponibles (figure 17). Les valeurs de cet indice, qui peuvent excéder $20^{\circ} \mathrm{C}$ à basse altitude (record d'ATD pour Mouterhouse avec $22,1^{\circ} \mathrm{C}$ ) tendent à décroître avec l'altitude.

Figure 17 : Distribution régionale de l'amplitude thermique diurne moyenne au cours de la période caniculaire (du 01 au 15 août 2003).

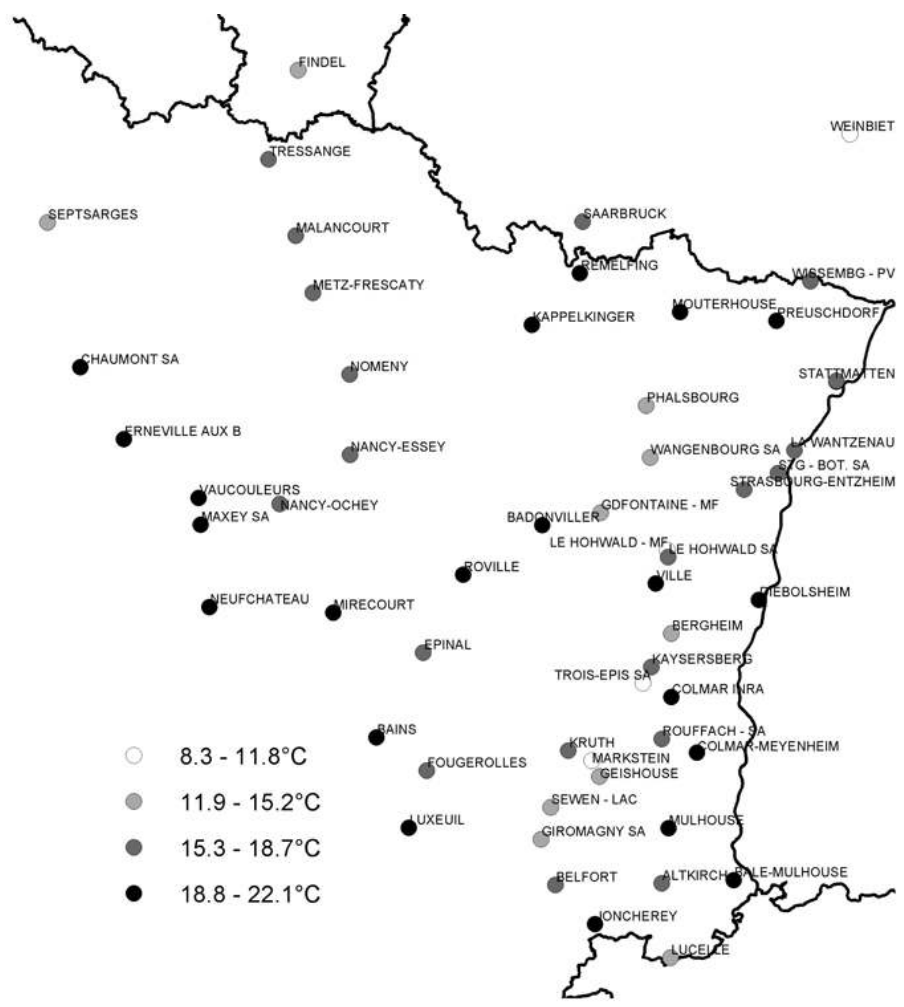

Le minimum est enregistré au Markstein avec $8,3^{\circ} \mathrm{C}$, ce qui explique que les nuits soient restées aussi douces en moyenne montagne pendant la période caniculaire. Par ailleurs, les sites mieux ventilés la nuit (plateau) ou caractérisés par des conditions de faible ventilation typiques des vallées à fond plat des Basses-Vosges propices à l'accumulation d'air frais (ex. Mouterhouse), ont été généralement affectés par les valeurs de Tn les plus faibles à l'échelle régionale. Ainsi, le 06 août 2003, la station forestière et lacustre de moyenne montagne de Kruth a connu la nuit la plus fraîche avec $15^{\circ} \mathrm{C}$ contre $21,5^{\circ} \mathrm{C}$ à la station de Grandfontaine SA à même altitude (figure 15). Dans le sillage des valeurs maximales des Tn du 06 août 2003, notons que le nombre de nuits tropicales a été exceptionnellement élevé au cours de l'année 2003 (figure 18a). 
Figure 18a :Distribution régionale du nombre de nuits tropicales $\left(\mathrm{Tn}>20^{\circ} \mathrm{C}\right)$ au cours de l'année 2003.

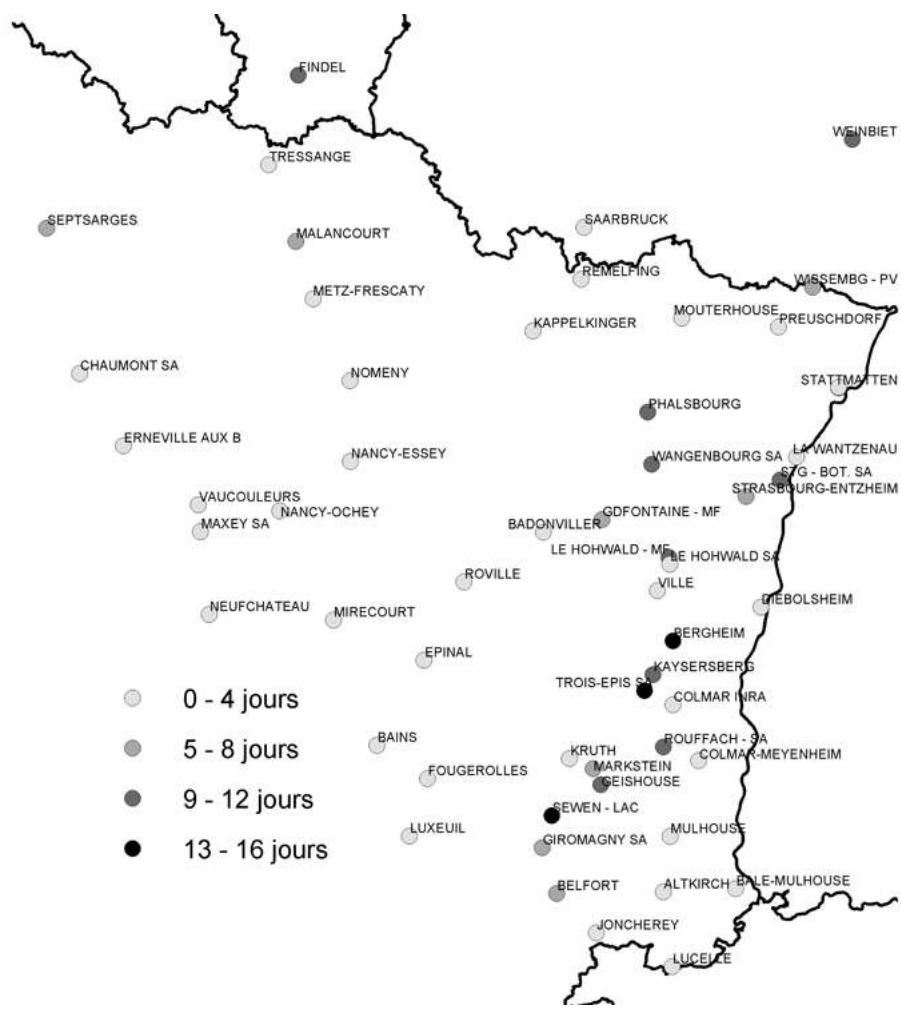

Figure 18b :Distribution régionale du nombre de jours de forte chaleur $\left(\mathrm{Tx}>30^{\circ} \mathrm{C}\right)$ au cours de l'année 2003.

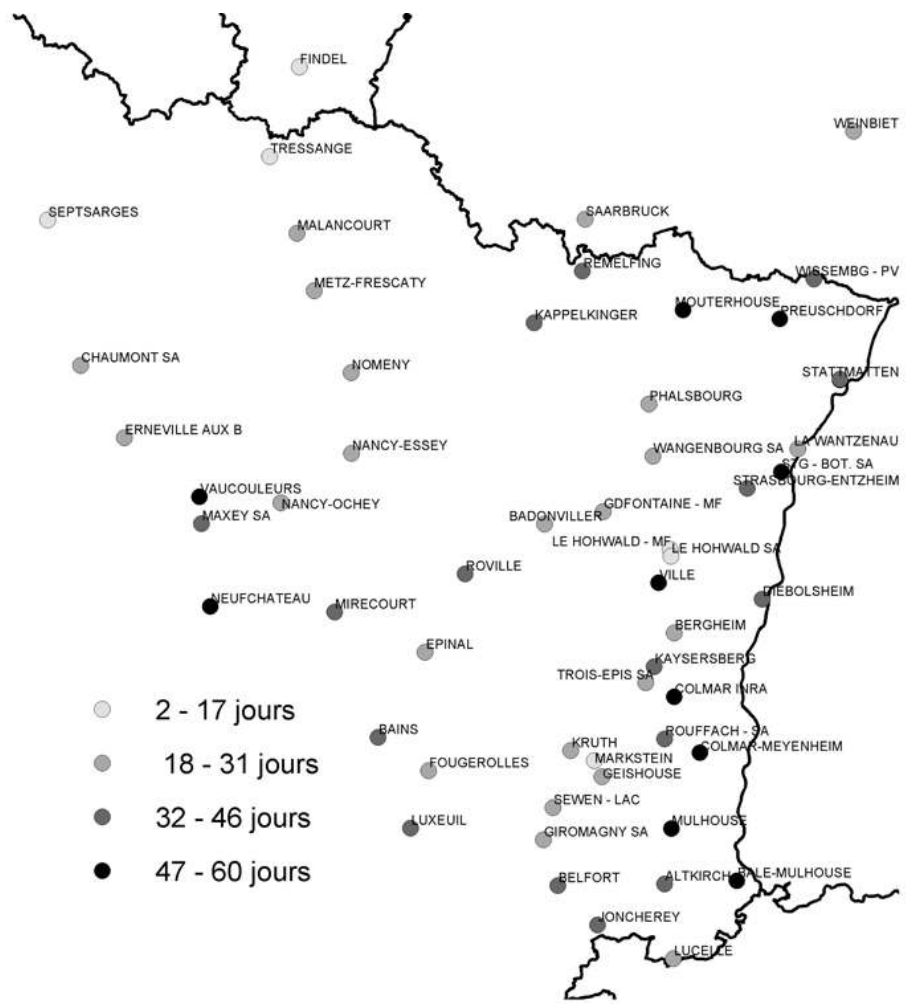



sud de la plaine d'Alsace et sur les reliefs, qui apparaissent en général après le franchissement du seuil de $30^{\circ} \mathrm{C}$. Elles ne constituent donc pas un facteur discriminant majeur entre les stations de mesure à l'échelle régionale. La répartition spatiale des jours de forte chaleur (figure 18b) montre que les occurrences dépassant 49 jours de forte chaleur, concernent la partie supérieure de la plaine d'Alsace (ex. ColmarMeyenheim, Bâle-Mulhouse) et quelques stations isolées comme Preuschdorf qui détient le record absolu avec 60 jours de forte chaleur. Deux axes parallèles aux antipodes de la région d'étude se dégagent ensuite totalisant plus de 38 jours de forte chaleur : un axe Kappelkinger-Stattmatten au nord et un axe Vaucouleurs-Joncherey au sud, qui confirment dans une certaine mesure les observations faites plus haut à propos des anomalies estivales affectant les Tx. Les stations de mesure situées plus en altitude enregistrent logiquement un nombre de jours très chauds moins élevé.

\section{Conclusion}

L'analyse des séries quotidiennes de températures extrêmes mesurées par 56 stations réparties dans le Nord-Est français et ses bordures luxembourgeoise et allemande a permis de documenter les anomalies annuelles et estivales de température au cours de l'année 2003 dans cette région, exceptionnellement chaude avec un écart de $1,5^{\circ} \mathrm{C}$ en moyenne régionale par rapport à la normale climatique 1971-2000. Le fait saillant qui ressort de cette étude réside dans la modulation importante des anomalies de température en fonction des multiples effets de site, et singulièrement des anomalies ayant affecté les températures minimales des mois de juin et d'août.

Une meilleure lisibilité du signal thermique lié à ces anomalies est perceptible aux stations de mesure de moyenne montagne, situées au-delà de $500 \mathrm{~m}$ d'altitude,

Revue Géographique de l'Est, vol. 45 / 2 | 2005 
échappant notamment aux inversions thermiques et aux conditions atmosphériques de basses couches des zones de basse altitude, où est amorti le signal thermique lié aux situations anticycloniques exceptionnellement persistantes qui ont marqué l'année 2003.

La magnitude des écarts de température aux moyennes décennales au cours de l'été 2003, laisse donc transparaître une dépendance altitudinale des anomalies de température extrêmes qui semblent augmenter linéairement avec l'altitude. En d'autres termes, en regard de l'élévation des températures qui semble s'accélérer au cours des quinze dernières années, les espaces montagnards du Nord-Est français et du Palatinat paraissent particulièrement vulnérables. Les stations de mesure en sommet de CLA (Couche-Limite Atmosphérique) ou représentatives de l'atmosphère libre, telles que la station du Markstein dans les Hautes-Vosges permettent de mieux détecter l'ampleur des anomalies de température à l'échelle saisonnière et annuelle.

Elles constitueront donc des sites de référence dans la région étudiée, pour détecter le réchauffement climatique attendu au cours des prochaines décennies sous l'effet, notamment, des émissions de gaz à effet de serre. Les travaux futurs tenteront justement d'affiner l'influence de l'effet de site (sites de fond de vallée par rapport aux sites de versant) et de la nature des sols (conductivité thermique, albédo) sur l'amplitude des anomalies thermiques à l'aide de transects topographiques représentatifs.

41 Il conviendrait également de mobiliser d'autres données micro-climatiques et singulièrement des données de vent qui permettraient de mieux comprendre la distribution spatiale des anomalies thermiques à l'échelle locale. Par ailleurs, l'année 2003 étant selon toute vraisemblance un avant-goût des événements climatiques récurrents d'ici la fin du présent siècle, une autre perspective de travail aura pour objectif de vérifier l'aptitude des modèles climatiques de méso-échelle à reproduire le découplage topoclimatique des anomalies de température entre les zones de faible altitude et de moyenne montagne.

\section{BIBLIOGRAPHIE}

BÉGUIN M., PUMAIN D. (2000). - La représentation des données géographiques : statistique et cartographie, Paris, Armand Colin, $192 \mathrm{p}$.

BENISTON M., REBETEZ M. (1996). - « Regional behaviour of minimum temperatures in Switzerland for the period 1979-1993», Theoretical and Applied Climatology, n 53, p. 231-243.

BENISTON M. (2004). — « The 2003 heat wave in Europe : a shape of things to come? An analysis based on Swiss climatological data and model simulations ", Geophysical Research Letters, $n^{\circ} 31$, L02202.

BESSEMOULIN P., BOURDETTE N., COURTIER P., MANACH J. (2004). — « La canicule d'août 2003 en France et en Europe ", La Météorologie, n 46, p. 25-33. 
FURY J., JOLY D. (1995). — « Interpolation spatiale à maille fine des températures journalières », La Météorologie, $\mathrm{n}^{\circ}$ 11, p. 36-43.

HANSEN J., RUEDY R., SATO M., IMHOFF M., LAWRENCE W., EASTERLING D., PETERSON T., KARL T. (2001). — « A closer look at United States and global surface temperture change ", Journal of Geophysical Research, n 106, p. 23 947-23963.

IMHOFF M.L., LAWRENCE W.T., STUTZER D.C., ELVIDGE C.D. (1997). — « A technique for using composite DMSP/OLS « city lights » satellite data to map urban area », Remote Sensing of Environment, $\mathrm{n}^{\circ}$ 61, p. 361-370.

KASTENDEUCH P. (1996). - Analyse à plusieurs échelles et modélisation des régimes de vents d'été dans le Fossé Rhénan méridional, Thèse de doctorat, Strasbourg, Université Louis Pasteur, 175 p.

OKE T.R. (1987). - Boundary layer climate, Londres, Methuen, $2^{\mathrm{e}}$ édition, $435 \mathrm{p}$.

PAUL P. (1997). - « Topoclimat dans le domaine tempéré semi-océanique ». In : Dubreuil et Marchand (éd.), Le climat, l'eau et les hommes, Rennes, Presses Universitaires de Rennes, p. 197-226. SCHÄR C., VIDALE P.L., LÜTHI D., FREI C., HÄBERLI C., LINIGER M., APPENZELLER C. (2004). - « The role of increasing temperature variability in European summer heat waves », Nature, doi : 10.1038/ nature02300.

WACKERNAGEL H. (1998). - Multivariate Geostatistics : an introduction with applications, Berlin, Springer, $2^{\mathrm{e}}$ édition, $291 \mathrm{p}$.

\section{ANNEXES}

Annexe A : Liste des stations de mesure climatologiques.

\begin{tabular}{|l|l|l|l|l|l|l|l|}
\hline INSEE & Nom & Ouverture & Type & Altitude & Latitude & Longitude & Producteur \\
\hline 54040001 & $\begin{array}{l}\text { BADONVILLER } \\
\text { (BADONVILLER-ONF) }\end{array}$ & $01 / 01 / 1929$ & 4 & 332 & $48^{\circ} 29^{\prime} 42^{\prime \prime} \mathrm{N}$ & $06^{\circ} 54^{\prime} 12^{\prime \prime} \mathrm{E}$ & METEO-FRANCE \\
\hline 54400001 & NOMENY & $01 / 01 / 1961$ & 4 & 208 & $48^{\circ} 52^{\prime} 54^{\prime \prime} \mathrm{N}$ & $06^{\circ} 14^{\prime} 06^{\prime \prime} \mathrm{E}$ & DIR. REG. DE L'ENV. \\
\hline 54405001 & OCHEY (NANCY-OCHEY) & $01 / 01 / 1963$ & 0 & 336 & $48^{\circ} 34^{\prime} 42^{\prime \prime} \mathrm{N}$ & $05^{\circ} 57^{\prime} 30^{\prime \prime} \mathrm{E}$ & METEO-FRANCE \\
\hline 54526001 & $\begin{array}{l}\text { TOMBLAINE (NANCY- } \\
\text { ESSEY) }\end{array}$ & $01 / 03 / 1921$ & 0 & 212 & $48^{\circ} 41^{\prime} 18^{\prime \prime} \mathrm{N}$ & $06^{\circ} 13^{\prime} 18^{\prime \prime} \mathrm{E}$ & METEO-FRANCE \\
\hline 55108003 & $\begin{array}{l}\text { CHAUMONT-SUR-AIRE } \\
\text { (CHAUMONT SA) }\end{array}$ & $01 / 09 / 1989$ & 2 & 240 & $48^{\circ} 55^{\prime} 36^{\prime \prime} \mathrm{N}$ & $05^{\circ} 15^{\prime} 18^{\prime \prime} \mathrm{E}$ & METEO-FRANCE \\
\hline 55179001 & $\begin{array}{l}\text { ERNEVILLE-AUX-BOIS } \\
\text { (ERNEVILLE AUX B) }\end{array}$ & $01 / 01 / 1932$ & 2 & 313 & $48^{\circ} 45^{\prime} 00^{\prime \prime} \mathrm{N}$ & $05^{\circ} 24^{\prime} 12^{\prime \prime} \mathrm{E}$ & METEO-FRANCE \\
\hline 55328002 & $\begin{array}{l}\text { MAXEY-SUR-VAISE (MAXEY } \\
\text { SA) }\end{array}$ & $01 / 06 / 1989$ & 2 & 250 & $48^{\circ} 32^{\prime} 12^{\prime \prime} \mathrm{N}$ & $05^{\circ} 40^{\prime} 12^{\prime \prime} \mathrm{E}$ & METEO-FRANCE \\
\hline
\end{tabular}




\begin{tabular}{|c|c|c|c|c|c|c|c|}
\hline 55484001 & SEPTSARGES & $01 / 09 / 1990$ & 2 & 293 & $49^{\circ} 16^{\prime} 36^{\prime \prime} \mathrm{N}$ & $05^{\circ} 09^{\prime} 18^{\prime \prime} \mathrm{E}$ & METEO-FRANCE \\
\hline 55533001 & VAUCOULEURS & $01 / 01 / 1950$ & 4 & 294 & $48^{\circ} 36^{\prime} 06^{\prime \prime} \mathrm{N}$ & $05^{\circ} 40^{\prime} 00^{\prime \prime} \mathrm{E}$ & METEO-FRANCE \\
\hline 57019001 & $\begin{array}{l}\text { AMNEVILLE } \\
\text { (MALANCOURT) }\end{array}$ & $01 / 10 / 1948$ & 4 & 350 & $49^{\circ} 13^{\prime} 12^{\prime \prime} \mathrm{N}$ & $06^{\circ} 03^{\prime} 48^{\prime \prime} \mathrm{E}$ & METEO-FRANCE \\
\hline 57039001 & AUGNY (METZ-FRESCATY) & $01 / 12 / 1929$ & 0 & 190 & $49^{\circ} 04^{\prime} 54^{\prime \prime} \mathrm{N}$ & $06^{\circ} 06^{\prime} 54^{\prime \prime} \mathrm{E}$ & METEO-FRANCE \\
\hline 57168001 & $\begin{array}{l}\text { DANNE-ET-QUATRE-VENTS } \\
\text { (PHALSBOURG) }\end{array}$ & $01 / 09 / 1945$ & 2 & 377 & $48^{\circ} 46^{\prime} 00^{\prime \prime} \mathrm{N}$ & $07^{\circ} 18^{\prime} 24^{\prime \prime} \mathrm{E}$ & METEO-FRANCE \\
\hline 57357001 & KAPPELKINGER & $01 / 12 / 1951$ & 4 & 223 & $48^{\circ} 58^{\prime} 36^{\prime \prime} \mathrm{N}$ & $06^{\circ} 54^{\prime} 30^{\prime \prime} \mathrm{E}$ & METEO-FRANCE \\
\hline 57489001 & MOUTERHOUSE & 01/02/1970 & 4 & 270 & $48^{\circ} 59^{\prime} 06^{\prime \prime} \mathrm{N}$ & $07^{\circ} 27^{\prime} 06^{\prime \prime} \mathrm{E}$ & METEO-FRANCE \\
\hline 57568001 & REMELFING & 01/08/1970 & 4 & 200 & $49^{\circ} 05^{\prime} 36^{\prime \prime} \mathrm{N}$ & $07^{\circ} 05^{\prime} 42^{\prime \prime} \mathrm{E}$ & METEO-FRANCE \\
\hline 57678001 & TRESSANGE & $01 / 01 / 1965$ & 4 & 358 & $49^{\circ} 24^{\prime} 24^{\prime \prime} \mathrm{N}$ & $05^{\circ} 58^{\prime} 54^{\prime \prime} \mathrm{E}$ & METEO-FRANCE \\
\hline 67090001 & DIEBOLSHEIM & 01/01/1986 & 4 & 162 & $48^{\circ} 17^{\prime} 00^{\prime \prime} \mathrm{N}$ & $07^{\circ} 40^{\prime} 00^{\prime \prime} \mathrm{E}$ & METEO-FRANCE \\
\hline 67122001 & $\begin{array}{l}\text { WANGENBOURG- } \\
\text { ENGENTHAL } \\
\text { (WANGENBOURG SA) }\end{array}$ & 01/05/1990 & 2 & 465 & $48^{\circ} 38^{\prime} 30^{\prime \prime} \mathrm{N}$ & $07^{\circ} 18^{\prime} 30^{\prime \prime} \mathrm{E}$ & AUTRES \\
\hline 67124001 & $\begin{array}{l}\text { ENTZHEIM (STRASBOURG- } \\
\text { ENTZHEIM) }\end{array}$ & $01 / 01 / 1921$ & 0 & 150 & $48^{\circ} 33^{\prime} 00^{\prime \prime} \mathrm{N}$ & $07^{\circ} 38^{\prime} 24^{\prime \prime} \mathrm{E}$ & METEO-FRANCE \\
\hline 67165003 & $\begin{array}{l}\text { GRANDFONTAINE } \\
\text { (GDFONTAINE - MF) }\end{array}$ & $01 / 08 / 1980$ & 4 & 515 & $48^{\circ} 31^{\prime} 00^{\prime \prime} \mathrm{N}$ & $07^{\circ} 07^{\prime} 00^{\prime \prime} \mathrm{E}$ & METEO-FRANCE \\
\hline 67210001 & $\begin{array}{l}\text { LE HOHWALD (LE } \\
\text { HOHWALD - MF) }\end{array}$ & $01 / 12 / 1935$ & 4 & 775 & $48^{\circ} 25^{\prime} 00^{\prime \prime} \mathrm{N}$ & $07^{\circ} 21^{\prime} 00^{\prime \prime} \mathrm{E}$ & METEO-FRANCE \\
\hline 67210002 & $\begin{array}{l}\text { LE HOHWALD (LE } \\
\text { HOHWALD SA) }\end{array}$ & $01 / 05 / 1990$ & 2 & 595 & $48^{\circ} 24^{\prime} 00^{\prime \prime} \mathrm{N}$ & $07^{\circ} 19^{\prime} 48^{\prime \prime} \mathrm{E}$ & AUTRES \\
\hline 67379001 & PREUSCHDORF & 01/01/1961 & 4 & 190 & $48^{\circ} 56^{\prime} 54^{\prime \prime} \mathrm{N}$ & $07^{\circ} 48^{\prime} 00^{\prime \prime} \mathrm{E}$ & METEO-FRANCE \\
\hline 67476001 & STATTMATTEN & $01 / 01 / 1947$ & 4 & 120 & $48^{\circ} 47^{\prime} 36^{\prime \prime} \mathrm{N}$ & $08^{\circ} 00^{\prime} 06^{\prime \prime} \mathrm{E}$ & METEO-FRANCE \\
\hline 67476002 & $\begin{array}{l}\text { STATTMATTEN } \\
\text { (STATTMATTEN SA) }\end{array}$ & 01/10/1986 & 2 & 121 & $48^{\circ} 47^{\prime} 42^{\prime \prime} \mathrm{N}$ & $08^{\circ} 00^{\prime} 06^{\prime \prime} \mathrm{E}$ & AUTRES \\
\hline 67482001 & $\begin{array}{l}\text { STRASBOURG (STG - BOT. } \\
\text { SA) }\end{array}$ & $01 / 03 / 1923$ & 2 & 139 & $48^{\circ} 35^{\prime} 00^{\prime \prime} \mathrm{N}$ & $07^{\circ} 46^{\prime} 00^{\prime \prime} \mathrm{E}$ & $\begin{array}{l}\text { INST. DE LA PHYS. } \\
\text { DU GLOBE }\end{array}$ \\
\hline 67507003 & VILLE & $01 / 10 / 1989$ & 4 & 255 & $48^{\circ} 20^{\prime} 18^{\prime \prime} \mathrm{N}$ & $07^{\circ} 18^{\prime} 00^{\prime \prime} \mathrm{E}$ & METEO-FRANCE \\
\hline 67519001 & $\begin{array}{l}\text { LA WANTZENAU (LA } \\
\text { WANTZENAU SA) }\end{array}$ & 01/03/1990 & 2 & 136 & $48^{\circ} 38^{\prime} 06^{\prime \prime} \mathrm{N}$ & $07^{\circ} 49^{\prime} 54^{\prime \prime} \mathrm{E}$ & AUTRES \\
\hline
\end{tabular}




\begin{tabular}{|c|c|c|c|c|c|c|c|}
\hline 67544003 & $\begin{array}{l}\text { WISSEMBOURG (WISSEMBG } \\
\text { - PV) }\end{array}$ & $01 / 01 / 1970$ & 4 & 172 & $49^{\circ} 02^{\prime} 18^{\prime \prime} \mathrm{N}$ & $07^{\circ} 56^{\prime} 00^{\prime \prime} \mathrm{E}$ & METEO-FRANCE \\
\hline 68004001 & ALTKIRCH & 01/01/1942 & 2 & 350 & $47^{\circ} 37^{\prime} 06^{\prime \prime} \mathrm{N}$ & $07^{\circ} 15^{\prime} 12^{\prime \prime} \mathrm{E}$ & METEO-FRANCE \\
\hline 68028001 & BERGHEIM & 01/03/1987 & 2 & 309 & $48^{\circ} 13^{\prime} 00^{\prime \prime} \mathrm{N}$ & $07^{\circ} 20^{\prime} 42^{\prime \prime} \mathrm{E}$ & $\begin{array}{l}\text { INST. NAT. DE LA } \\
\text { RECH. AGR. }\end{array}$ \\
\hline 68066001 & COLMAR (COLMAR INRA) & 01/01/1949 & 2 & 202 & $48^{\circ} 03^{\prime} 48^{\prime \prime} \mathrm{N}$ & $07^{\circ} 19^{\prime} 48^{\prime \prime} \mathrm{E}$ & $\begin{array}{l}\text { INST. NAT. DE LA } \\
\text { RECH. AGR. }\end{array}$ \\
\hline 68102001 & GEISHOUSE & 01/01/1964 & 2 & 660 & $47^{\circ} 53^{\prime} 00^{\prime \prime} \mathrm{N}$ & $07^{\circ} 03^{\prime} 18^{\prime \prime} \mathrm{E}$ & METEO-FRANCE \\
\hline 68162001 & KAYSERSBERG & 01/01/1900 & 4 & 248 & $48^{\circ} 08^{\prime} 18^{\prime \prime} \mathrm{N}$ & $07^{\circ} 15^{\prime} 54^{\prime \prime} \mathrm{E}$ & METEO-FRANCE \\
\hline 68171002 & KRUTH & 01/01/1961 & 4 & 512 & $47^{\circ} 57^{\prime} 00^{\prime \prime} \mathrm{N}$ & $06^{\circ} 57^{\prime} 00^{\prime \prime} \mathrm{E}$ & $\begin{array}{l}\text { DIR. DEP. DE } \\
\text { L'AGRICULTURE }\end{array}$ \\
\hline 68190001 & LUCELLE & 01/04/1987 & 2 & 690 & $47^{\circ} 26^{\prime} 18^{\prime \prime} \mathrm{N}$ & $07^{\circ} 16^{\prime} 06^{\prime \prime} \mathrm{E}$ & METEO-FRANCE \\
\hline 68205001 & $\begin{array}{l}\text { MEYENHEIM (COLMAR- } \\
\text { MEYENHEIM) }\end{array}$ & 01/09/1956 & 0 & 211 & $47^{\circ} 55^{\prime} 36^{\prime \prime} \mathrm{N}$ & $07^{\circ} 24^{\prime} 30^{\prime \prime} \mathrm{E}$ & METEO-FRANCE \\
\hline 68224006 & MULHOUSE & 01/05/1953 & 2 & 245 & $47^{\circ} 45^{\prime} 00^{\prime \prime} \mathrm{N}$ & $07^{\circ} 17^{\prime} 24^{\prime \prime} \mathrm{E}$ & METEO-FRANCE \\
\hline 68247003 & $\begin{array}{l}\text { ODEREN (MARKSTEIN } \\
\text { CRETE) }\end{array}$ & $01 / 01 / 1989$ & 2 & 1184 & $47^{\circ} 55^{\prime} 24^{\prime \prime} \mathrm{N}$ & $07^{\circ} 01^{\prime} 54^{\prime \prime} \mathrm{E}$ & METEO-FRANCE \\
\hline 68287003 & $\begin{array}{l}\text { ROUFFACH (ROUFFACH - } \\
\text { SA) }\end{array}$ & $01 / 10 / 1986$ & 2 & 285 & $47^{\circ} 57^{\prime} 54^{\prime \prime} \mathrm{N}$ & $07^{\circ} 17^{\prime} 12^{\prime \prime} \mathrm{E}$ & METEO-FRANCE \\
\hline 68297001 & $\begin{array}{l}\text { SAINT-LOUIS (BALE- } \\
\text { MULHOUSE) }\end{array}$ & $01 / 08 / 1946$ & 0 & 263 & $47^{\circ} 36^{\prime} 54^{\prime \prime} \mathrm{N}$ & $07^{\circ} 30^{\prime} 36^{\prime \prime} \mathrm{E}$ & METEO-FRANCE \\
\hline 68307001 & SEWEN (SEWEN - LAC) & $01 / 11 / 1888$ & 4 & 620 & $47^{\circ} 49^{\prime} 00^{\prime \prime} \mathrm{N}$ & $06^{\circ} 52^{\prime} 30^{\prime \prime} \mathrm{E}$ & METEO-FRANCE \\
\hline 68338001 & $\begin{array}{l}\text { TURCKHEIM (TROIS-EPIS } \\
\text { SA) }\end{array}$ & $01 / 07 / 1985$ & 2 & 660 & $48^{\circ} 06^{\prime} 06^{\prime \prime} \mathrm{N}$ & $07^{\circ} 13^{\prime} 54^{\prime \prime} \mathrm{E}$ & CONSEIL GENERAL \\
\hline 88029001 & BAINS-LES-BAINS (BAINS) & $01 / 05 / 1949$ & 4 & 316 & $48^{\circ} 00^{\prime} 24^{\prime \prime} \mathrm{N}$ & $06^{\circ} 16^{\prime} 00^{\prime \prime} \mathrm{E}$ & METEO-FRANCE \\
\hline 88136001 & DOGNEVILLE (EPINAL) & $01 / 06 / 1986$ & 1 & 320 & $48^{\circ} 12^{\prime} 18^{\prime \prime} \mathrm{N}$ & $06^{\circ} 27^{\prime} 00^{\prime \prime} \mathrm{E}$ & METEO-FRANCE \\
\hline 88304005 & MIRECOURT & 01/01/1939 & 4 & 265 & $48^{\circ} 18^{\prime} 42^{\prime \prime} \mathrm{N}$ & $06^{\circ} 08^{\prime} 00^{\prime \prime} \mathrm{E}$ & METEO-FRANCE \\
\hline 88321001 & NEUFCHÂTEAU & $01 / 01 / 1859$ & 4 & 286 & $48^{\circ} 20^{\prime} 18^{\prime \prime} \mathrm{N}$ & $05^{\circ} 41^{\prime} 18^{\prime \prime} \mathrm{E}$ & METEO-FRANCE \\
\hline 88402002 & $\begin{array}{l}\text { ROVILLE-AUX-CHENES } \\
\text { (ROVILLE) }\end{array}$ & 01/04/1950 & 2 & 280 & $48^{\circ} 23^{\prime} 12^{\prime \prime} \mathrm{N}$ & $06^{\circ} 36^{\prime} 30^{\prime \prime} \mathrm{E}$ & AUTRES \\
\hline 70245001 & FOUGEROLLES & 01/01/1957 & 4 & 473 & $47^{\circ} 55^{\prime} 18^{\prime \prime} \mathrm{N}$ & $06^{\circ} 26^{\prime} 24^{\prime \prime} \mathrm{E}$ & METEO-FRANCE \\
\hline
\end{tabular}




\begin{tabular}{|c|c|c|c|c|c|c|c|}
\hline 70473001 & SAINT-SAUVEUR (LUXEUIL) & 01/01/1918 & 0 & 271 & $47^{\circ} 47^{\prime} 12^{\prime \prime} \mathrm{N}$ & $06^{\circ} 21^{\prime} 54^{\prime \prime} \mathrm{E}$ & METEO-FRANCE \\
\hline 90010001 & BELFORT & 01/03/1946 & 1 & 422 & $47^{\circ} 37^{\prime} 48^{\prime \prime} \mathrm{N}$ & $06^{\circ} 52^{\prime} 36^{\prime \prime} \mathrm{E}$ & METEO-FRANCE \\
\hline 90052002 & $\begin{array}{l}\text { GIROMAGNY (GIROMAGNY } \\
\text { SA) }\end{array}$ & $01 / 07 / 1990$ & 2 & 473 & $47^{\circ} 44^{\prime} 30^{\prime \prime} \mathrm{N}$ & $06^{\circ} 50^{\prime} 06^{\prime \prime} \mathrm{E}$ & METEO-FRANCE \\
\hline \multirow[t]{4}{*}{90056002} & JONCHEREY & 01/12/1954 & 4 & 367 & $47^{\circ} 31^{\prime} 48^{\prime \prime} \mathrm{N}$ & $07^{\circ} 00^{\prime} 30^{\prime \prime} \mathrm{E}$ & METEO-FRANCE \\
\hline & FINDEL & 01/01/1947 & 1 & 380 & $49^{\circ} 39^{\prime} \mathrm{N}$ & $06^{\circ} 08^{\prime} \mathrm{E}$ & $\begin{array}{l}\text { AEROPORT DE } \\
\text { LUXEMBOURG }\end{array}$ \\
\hline & SAARBRUCK & 02/01/1951 & 1 & 320 & $49^{\circ} 13^{\prime} \mathrm{N}$ & $07^{\circ} 07^{\prime} \mathrm{E}$ & $\begin{array}{l}\text { DEUTSCHER } \\
\text { WETTERDIENST }\end{array}$ \\
\hline & WEINBIET & 03/01/1953 & 1 & 553 & $49^{\circ} 23^{\prime} \mathrm{N}$ & $08^{\circ} 07^{\prime} \mathrm{E}$ & $\begin{array}{l}\text { DEUTSCHER } \\
\text { WETTERDIENST }\end{array}$ \\
\hline & $\begin{array}{l}\text { données journalières disponi } \\
\text { uniquement sur l'année } 2003\end{array}$ & & 1 & $\begin{array}{l}\text { Stati } \\
\text { dista }\end{array}$ & noptique non & professionne & elle ou observation à \\
\hline & $\begin{array}{l}\text { données journalières disponi } \\
2003 \text { et données mensuelles }\end{array}$ & les sur l'année & 2 & Stati & utomatique, $t$ & mps réel sur & la période 1991-2000 \\
\hline & $\begin{array}{l}\text { données journalières disponi } \\
\text { période } 1971-2003\end{array}$ & les sur la & 3 & Stati & utomatique, $t \in$ & mps différé & \\
\hline 0 & $\begin{array}{l}\text { Station synoptique professior } \\
\text { observation sur place }\end{array}$ & Inelle, avec & 4 & Post & natologique & & \\
\hline
\end{tabular}

Annexe B : Caractéristiques de site des stations de mesure climatologiques.

\begin{tabular}{|l|l|l|l|l|}
\hline INSEE & Nom & Situation topographique & Exposition & Environnement \\
\hline 54040001 & BADONVILLER (BADONVILLER- \\
ONF) & $\begin{array}{l}\text { pied de pente/fond de } \\
\text { vallon }\end{array}$ & SW & rural \\
\hline 54400001 & NOMENY & plateau & - & rural \\
\hline 54405001 & OCHEY (NANCY-OCHEY) & plateau & - & aérodrome \\
\hline 54526001 & TOMBLAINE (NANCY-ESSEY) & pied de pente/fond de & W & urbain/eau \\
\hline 55108003 & $\begin{array}{l}\text { CHAUMONT-SUR-AIRE } \\
\text { (CHAUMONT SA) }\end{array}$ & $\begin{array}{l}\text { pied de pente/fond de } \\
\text { vallée }\end{array}$ & SW & sub-urbain \\
\hline
\end{tabular}




\begin{tabular}{|c|c|c|c|c|}
\hline 55179001 & $\begin{array}{l}\text { ERNEVILLE-AUX-BOIS } \\
\text { (ERNEVILLE AUX B) }\end{array}$ & mi-pente/vallée étroite & W & rural \\
\hline 55328002 & $\begin{array}{l}\text { MAXEY-SUR-VAISE (MAXEY } \\
\text { SA) }\end{array}$ & plaine & E & rural \\
\hline 55484001 & SEPTSARGES & sommet de pente & $\mathrm{NE}$ & rural \\
\hline 55533001 & VAUCOULEURS & pied de pente/cuesta & E & sub-urbain \\
\hline 57019001 & AMNEVILLE (MALANCOURT) & plateau & SE & urbain lâche \\
\hline 57039001 & AUGNY (METZ-FRESCATY) & plaine & - & $\begin{array}{l}\text { aérodrome/nombreux } \\
\text { plans d'eau }\end{array}$ \\
\hline 57168001 & $\begin{array}{l}\text { DANNE-ET-QUATRE-VENTS } \\
\text { (PHALSBOURG) }\end{array}$ & plateau & W & rural \\
\hline 57357001 & KAPPELKINGER & mi-pente/vallée étroite & SW & rural \\
\hline 57489001 & MOUTERHOUSE & mi-pente/vallée évasée & SW & forestier/plan d'eau \\
\hline 57568001 & REMELFING & $\begin{array}{l}\text { pied de pente/fond de } \\
\text { vallée }\end{array}$ & SW & sub-urbain/eau \\
\hline 57678001 & TRESSANGE & plateau & SE & rural \\
\hline 67090001 & DIEBOLSHEIM & plaine & - & rural/eau \\
\hline 67122001 & $\begin{array}{l}\text { WANGENBOURG-ENGENTHAL } \\
\text { (WANGENBOURG SA) }\end{array}$ & sommet de pente & E & rural/forestier \\
\hline 67124001 & $\begin{array}{l}\text { ENTZHEIM (STRASBOURG- } \\
\text { ENTZHEIM) }\end{array}$ & plaine & - & rural \\
\hline 67165003 & \begin{tabular}{|l} 
GRANDFONTAINE \\
(GDFONTAINE - MF)
\end{tabular} & $\begin{array}{l}\text { mi-pente/vallée étroite } \\
\text { et profonde }\end{array}$ & SE & forestier \\
\hline 67210001 & $\begin{array}{l}\text { LE HOHWALD (LE HOHWALD - } \\
\text { MF) }\end{array}$ & $\begin{array}{l}\text { pied de pente/vallée } \\
\text { étroite et profonde }\end{array}$ & S & forestier \\
\hline 67210002 & $\begin{array}{l}\text { LE HOHWALD (LE HOHWALD } \\
\text { SA) }\end{array}$ & $\begin{array}{l}\text { mi-pente/vallée étroite } \\
\text { et profonde }\end{array}$ & $\mathrm{N}$ & forestier \\
\hline 67379001 & PREUSCHDORF & $\begin{array}{l}\text { pied de pente/fond de } \\
\text { vallée évasée }\end{array}$ & SW & rural \\
\hline 67476001 & STATTMATTEN & plaine & - & rural/eau \\
\hline 67476002 & $\begin{array}{l}\text { STATTMATTEN } \\
\text { (STATTMATTEN SA) }\end{array}$ & plaine & - & rural/eau \\
\hline
\end{tabular}




\begin{tabular}{|c|c|c|c|c|}
\hline 67482001 & STRASBOURG (STG - BOT. SA) & plaine & - & urbain dense \\
\hline 67507003 & VILLE & $\begin{array}{l}\text { pied de pente/fond de } \\
\text { vallée }\end{array}$ & $\mathrm{N}$ & rural \\
\hline 67519001 & $\begin{array}{l}\text { LA WANTZENAU (LA } \\
\text { WANTZENAU SA) }\end{array}$ & plaine & - & rural/eau \\
\hline 67544003 & $\begin{array}{l}\text { WISSEMBOURG (WISSEMBG - } \\
\text { PV) }\end{array}$ & $\begin{array}{l}\text { pied de pente/fond de } \\
\text { vallée évasée }\end{array}$ & $\mathrm{N}$ & sub-urbain \\
\hline 68004001 & ALTKIRCH & sommet de pente & $\mathrm{N}$ & rural \\
\hline 68028001 & BERGHEIM & $\begin{array}{l}\text { mi-pente/vallée étroite } \\
\text { et profonde }\end{array}$ & SE & rural \\
\hline 68066001 & COLMAR (COLMAR INRA) & plaine & - & rural \\
\hline 68102001 & GEISHOUSE & $\begin{array}{l}\text { mi-pente/vallée étroite } \\
\text { et profonde }\end{array}$ & SE & rural \\
\hline 68162001 & KAYSERSBERG & $\begin{array}{l}\text { pied de pente/vallée } \\
\text { étroite et profonde }\end{array}$ & S & urbain \\
\hline 68171002 & KRUTH & $\begin{array}{l}\text { pied de pente/vallée } \\
\text { étroite et profonde }\end{array}$ & E & forestier/plan d'eau \\
\hline 68190001 & LUCELLE & $\begin{array}{l}\text { mi-pente/vallée étroite } \\
\text { et profonde }\end{array}$ & SE & rural \\
\hline 68205001 & $\begin{array}{l}\text { MEYENHEIM (COLMAR- } \\
\text { MEYENHEIM) }\end{array}$ & plaine & - & aérodrome \\
\hline 68224006 & MULHOUSE & plaine & - & urbain lâche/eau \\
\hline 68247003 & ODEREN (MARKSTEIN CRETE) & crête & - & rural \\
\hline 68287003 & ROUFFACH (ROUFFACH - SA) & mi-pente/piedmont & E & rural \\
\hline 68297001 & $\begin{array}{l}\text { SAINT-LOUIS (BALE- } \\
\text { MULHOUSE) }\end{array}$ & plaine & - & aéroport \\
\hline 68307001 & SEWEN (SEWEN - LAC) & $\begin{array}{l}\text { pied de pente/vallée } \\
\text { étroite et profonde }\end{array}$ & SE & forestier/eau \\
\hline 68338001 & TURCKHEIM (TROIS-EPIS SA) & $\begin{array}{l}\text { pied de pente/vallée } \\
\text { étroite et profonde }\end{array}$ & SE & rural \\
\hline 88029001 & BAINS-LES-BAINS (BAINS) & $\begin{array}{l}\text { pied de pente/fond de } \\
\text { cuvette }\end{array}$ & W & rural \\
\hline 88136001 & DOGNEVILLE (EPINAL) & pied de pente/terrasse & NW & rural \\
\hline
\end{tabular}




\begin{tabular}{|c|c|c|c|c|}
\hline 88304005 & MIRECOURT & $\begin{array}{l}\text { pied de pente/fond de } \\
\text { vallée }\end{array}$ & NW & rural/eau \\
\hline 88321001 & NEUFCHÂTEAU & sommet de pente & $\mathrm{N}$ & rural \\
\hline 88402002 & $\begin{array}{l}\text { ROVILLE-AUX-CHÊNES } \\
\text { (ROVILLE) }\end{array}$ & plaine & - & rural/eau \\
\hline 70245001 & FOUGEROLLES & sommet de pente & $\mathrm{S}$ & rural \\
\hline 70473001 & SAINT-SAUVEUR (LUXEUIL) & mi-pente/vallée évasée & NW & rural/clairière \\
\hline 90010001 & BELFORT & plaine & - & urbain lâche \\
\hline 90052002 & GIROMAGNY (GIROMAGNY SA) & plaine & W & urbain lâche \\
\hline 90056002 & JONCHEREY & mi-pente/vallée évasée & SW & rural \\
\hline & FINDEL & plateau & - & aéroport \\
\hline & SAARBRUCK & plaine & SW & urbain \\
\hline & WEINBIET & sommet de pente & $\mathrm{NE}$ & forestier \\
\hline
\end{tabular}

\section{RÉSUMÉS}

Les valeurs de températures extrêmes quotidiennes (Tn et Tx) enregistrées au cours de l'année 2003 sont analysées pour plus de cinquante stations de mesure climatologiques réparties sur le Nord-Est français et sur la bordure frontalière. Dans un premier temps les moyennes annuelles de températures extrêmes de l'année 2003 sont positionnées par rapport à la normale climatique 1971-2000 et la décennie 1991-2000, marquée par une succession d'années chaudes. L'épisode de chaleur estival est ensuite étudié à l'aide de différents indices de température dont la répartition spatiale met en évidence la vulnérabilité des espaces de moyenne montagne au réchauffement climatique en cours.

Daily values of extreme air temperatures (Tn and Tx) for the year 2003 were investigated for more than fifty weather stations distributed throughout northeastern France, including bordering areas. A first step was to compare mean annual values of extreme air temperatures to the 1971-2000 and the 1991-2000 baseline periods, the latter being affected by a sequence of warm years. The summer 2003 heat wave was next analysed, based on a set of temperature indices. The spatial distribution of these indices shows the vulnerability of middle mountains to the recent climate warming.

Extreme Tageslufttemperaturen (Tn und Tx) des Jahres 2003 sind in mehr als 50 Wetterbeobachtungsstationen im Nordosten Frankreichs, sowie in den Grenzgebieten, untersucht worden. Zuerst wurden die Jahresmittelwerte der extremen Lufttemperaturen mit den Normen der Perioden 1971-2000 und 1991-2000 verglichen, wobei letztere von einer Sequenz mit wärmeren Jahren gekennzeichnet ist. Anschliessend wurde die Hitzewelle des Sommers 2003 
mit Hilfe von verschiedenen Temperaturindikatoren analysiert. Die räumliche Verteilung dieser Indikatoren zeigt dass Mittelgebirge am stärksten von der rezenten Klimaerwärmung betroffen sind.

INDEX

Mots-clés : canicule, Nord-Est de la France, réchauffement climatique, température de l'air extrême

Schlüsselwörter : Extreme Tageslufttemperatur, Hitzewelle, Klimaerwärmung, Nordostfrankreich

Keywords : climate warming, extreme air temperature, heat wave, Northeastern France

\section{AUTEURS}

\section{GILLES DROGUE}

CREBS - Cellule de Recherche en Environnement et BiotechnologieS, Centre de Recherche Public - Gabriel Lippmann, 41 rue du Brill, L-4422 Belvaux, LUXEMBOURG, drogue@crpgl.lu

\section{LUCIEN HOFFMANN}

CREBS - Cellule de Recherche en Environnement et BiotechnologieS, Centre de Recherche Public

- Gabriel Lippmann, 41 rue du Brill, L-4422 Belvaux, LUXEMBOURG

\section{LAURENT PFISTER}

CREBS - Cellule de Recherche en Environnement et BiotechnologieS, Centre de Recherche Public - Gabriel Lippmann, 41 rue du Brill, L-4422 Belvaux, LUXEMBOURG

\section{PATRICE PAUL}

Institut de Géographie, Université Louis Pasteur, 3 rue de l'Argonne, F-67083 Strasbourg cedex, FRANCE, epaul@equinoxe.u-strasbg.fr 